\title{
Factors influencing the understandability of process models: A systematic literature review
}

\author{
Ahmet Dikici $^{\mathrm{a}, *}$, Oktay Turetken ${ }^{\mathrm{b}}$, Onur Demirors ${ }^{\mathrm{c}, \mathrm{d}}$ \\ a TUBITAK BILGEM Software Technologies Research Institute, Ankara, Turkey \\ ${ }^{\mathrm{b}}$ Eindhoven University of Technology, Eindhoven, Netherlands \\ ${ }^{c}$ Department of Computer Engineering, Izmir Institute of Technology, Izmir, Turkey \\ ${ }^{\mathrm{d}}$ School of Computer Science and Engineering, University of New South Wales, Sydney, Australia
}

\section{A R T I C L E I N F O}

\section{Article history:}

Received 28 October 2016

Revised 15 July 2017

Accepted 1 September 2017

Available online 6 September 2017

\section{Keywords:}

Business process model

Understandability

Comprehension

Process model understandability

Systematic literature review

\begin{abstract}
A B S T R A C T
Context: Process models are key in facilitating communication in organizations and in designing processaware information systems. Organizations are facing increasingly larger and more complex processes, which pose difficulties to the understandability of process models. The literature reports several factors that are considered to influence the understandability of process models. However, these studies typically focus on testing of a limited set of factors. A work that collects, abstracts and synthesizes an in-depth summary of the current literature will help in developing the research in this field.

Objective: We conducted a systematic literature review (SLR) focusing on the empirical studies in the existing literature in order to better understand the state of the research on process model understandability, and identify the gaps and opportunities for future research.

Method: We searched the studies between the years 1995 and 2015 in established electronic libraries. Out of 1066 publications retrieved initially, we selected 45 publications for thorough analysis. We identified, analyzed and categorized factors that are considered to influence the understandability of process models as studied in the literature using empirical methods. We also analyzed the indicators that are used to quantify process model understandability.

Results: Our analysis identifies several gaps in the field, as well as issues of inconsistent findings regarding the effect of some factors, unbalanced emphasis on certain indicators, and methodological concerns. Conclusions: The existing research calls for comprehensive empirical studies to contribute to a better understanding of the factors of process model understandability. Our study is a comprehensive source for researchers working on the understandability of process models and related fields, and a useful guide for practitioners aiming to generate understandable process models.
\end{abstract}

(C) 2017 Elsevier B.V. All rights reserved.

\section{Introduction}

Process modeling is a fundamental activity to understand and communicate process information, and often a prerequisite for conducting analysis, redesign and automation [1]. As such, process models are used for many purposes from increasing understanding of a process by knowledge workers, executing a process, sharing process information with customers, or for what-if analysis [2,3]. However, in order for these models to successfully serve for their potential uses, they should be understandable to their audience.

Process model understandability (or comprehension) can be defined as the degree to which information contained in a process

\footnotetext{
* Corresponding author.

E-mail addresses: ahmet.dikici@tubitak.gov.tr, ahmetdikici@gmail.com (A. Dikici), o.turetken@tue.nl (O. Turetken), demirorso@gmail.com (O. Demirors).
}

model can be easily understood by a reader of that model [4]. It is typically associated with the ease of use and the effort required for reading and correctly interpreting a process model [5]. It can be regarded as a learning process where the users of a process model integrate model content with their previous experience in order to construct new knowledge as an output of this learning process [6].

In general, model understandability is regarded one of the most important quality attributes of conceptual models. It is one of the essential components of conceptual modeling quality frameworks (such as the SEQUAL framework [7], Conceptual Modeling Quality Framework-CMQF [8]), and of the quality frameworks on business process models (such as the SIQ [9], 3QM [10, 11] and [12]). A common and major dimension of quality in all these mainstream frameworks is model's pragmatic quality, which relates to the understandability, i.e. whether a graphical model can be understood by its intended reader. 
Knowledge workers from various business units and technical departments are increasingly involved in the modeling of processes [13-15]. The number and diversity of process model designers has widely dispersed. On the other hand, lack of qualified process modelers is identified as one of the typical characteristics of unsuccessful business process management (BPM) projects, which causes quality issues that might adversely influence the understandability of process models [16]. Research on verification of process models shows that there are critical problems with their construction and understandability. For instance, many process model collections from practice have error rates of up to 20\% [17]. Therefore, process modelers should regard understandability as an important quality attribute to be achieved.

Creating understandable process models requires taking into account various factors (such as the size and complexity of the model) that are assumed to influence the understandability of process models. Knowledge on the effects of these factors on the understandability is important both for research and practice. Hence, several empirical studies have been performed to investigate the effect of these factors. However, each study investigates a limited set of certain factors, and reports findings which are sometimes contradictory to the conclusions of prior works. The research in this field lacks an overview of the factors influencing the understandability of process models. An extensive summary of the existing literature on this field would be a valuable source for researchers in the process modeling field, and for practitioners working with process models.

With the aim to better understand the state of the research on process model understandability, and to identify the gaps and opportunities for future research, we performed a systematic literature review (SLR). We collected and synthesized an in-depth summary of the current literature on the factors influencing the understandability of process models, and the way they are operationalized, as well as the indicators used to quantify process model understandability. In this paper, we focus on the visual process models such as those established using eEPC and BPMN notations.

SLRs have been performed on various topics of process modeling, some of which also relates to the understandability of process models. de Oca et al. [18] present an SLR conducted with the aim to determine the aspects of process modeling quality that had been addressed in the literature and gaps remained to be covered. Similarly, Gonzalez et al. [19] performed an SLR on business process measurement. The results of both studies indicate that most research in business process modeling focuses on model's pragmatic quality aspects, in particular on the understandability of process models. On a closely related and broader topic, Houy et al. [20] systematically reviewed how conceptual model understandability is measured in the literature, and proposed a framework covering different dimensions of model understandability. The results indicate a lack of consensus on what constitutes the central aspects and dimensions of model understandability within the conceptual modeling community. As a continuation of their research, Houy et al. [5] report on an SLR that aims to understand the extent by which the process model understandability research is multi-theoretically founded. The results indicate an increasing emphasis on the theoretical foundations in process model understandability research.

In addition to the above-mentioned works, a number of process modeling guidelines have been proposed to guide process modelers for higher model quality (for instance, Guidelines of Business Process Modeling [13], Seven Process Modeling Guidelines 7PMG [21], and Modeling Guidelines for Business Process Models [22]). However, an in-depth systematic literature review that concentrates on the factors of process model understandability and on the indicators operationalizing process model understandability has not been conducted so far.
Accordingly, we formulated the following research questions for our review.

RQ1. How is the understandability of process models operationalized in the literature?

Understanding the influence of factors on process model understandability requires -as a first step, a clear and explicit definition of the theoretical concept (i.e. the process model understandability), and the way it is operationalized. Various indicators have been proposed in the literature for operationalizing model understandability. Our first research question aims to provide a structured view and insight into this diversity.

RQ2. Which factors that are considered to influence the understandability of process models have been investigated in the literature?

The objective in the second research question is to investigate extensively the factors that have been studied in the literature.

The answers to these research questions are based on the findings of the studies that empirically tested the influence of a set of factors on process model understandability. Hence, in reviewing the existing literature, we elicited information from empirical works, which employed experimental methods that involve participants to test the effect of a set of factors that are assumed to influence the understandability of process models. We searched and examined the studies performed between the years 1995 and 2015 in a comprehensive set of academic digital libraries. We initially retrieved 1066 studies; 45 of which were finally selected in accordance to our selection procedure and criteria. We thoroughly analyzed their research design and applied methods. We investigated the factors that are tested, the way they are operationalized, the indicators used to quantify model understandability, and the key findings. The results of our review provide a significant input to the current body of knowledge in this field, which is of importance both to the researchers and practitioners.

The remaining of this paper is structured as follows. In Section 2, we present the design of our research and the method that we followed for the SLR. Section 3 provides an overview of general findings. Sections 4 and 5 summarize our findings obtained from the detailed analysis of selected publications that we performed to answer our research questions. In Section 6, we discuss our findings and the research gaps that we identified for future research. Finally, Section 7 concludes our paper.

\section{Research design}

In conducting this SLR, we followed the guidelines provided by Kitchenham and Charters [23] and Webster and Watson [24]. The literature search was performed for the studies published in the academic journals and conference proceedings between the years 1995 and 2015 (February). MS Excel was used to store publication information, calculate numerical results, prepare related charts, and communicate internally between the authors. The following steps, shown in Fig. 1, were derived from the guidelines for performing SLRs in software engineering [23] and applied as a procedure in systematically searching and selecting the relevant studies. In the rest of this section, we describe the details of these steps.

Step 1. Define the research problem: As a first step, we defined and stated our research problem.

Step 2. Define the research objective and questions: Based on our research problem, we defined the research objective and formulated the research questions.

Step 3. Conduct pilot searches: The third step was conducted to review the scope, try different searches, and see the different results in order to refine the search string to be used for the subsequent comprehensive searches. 


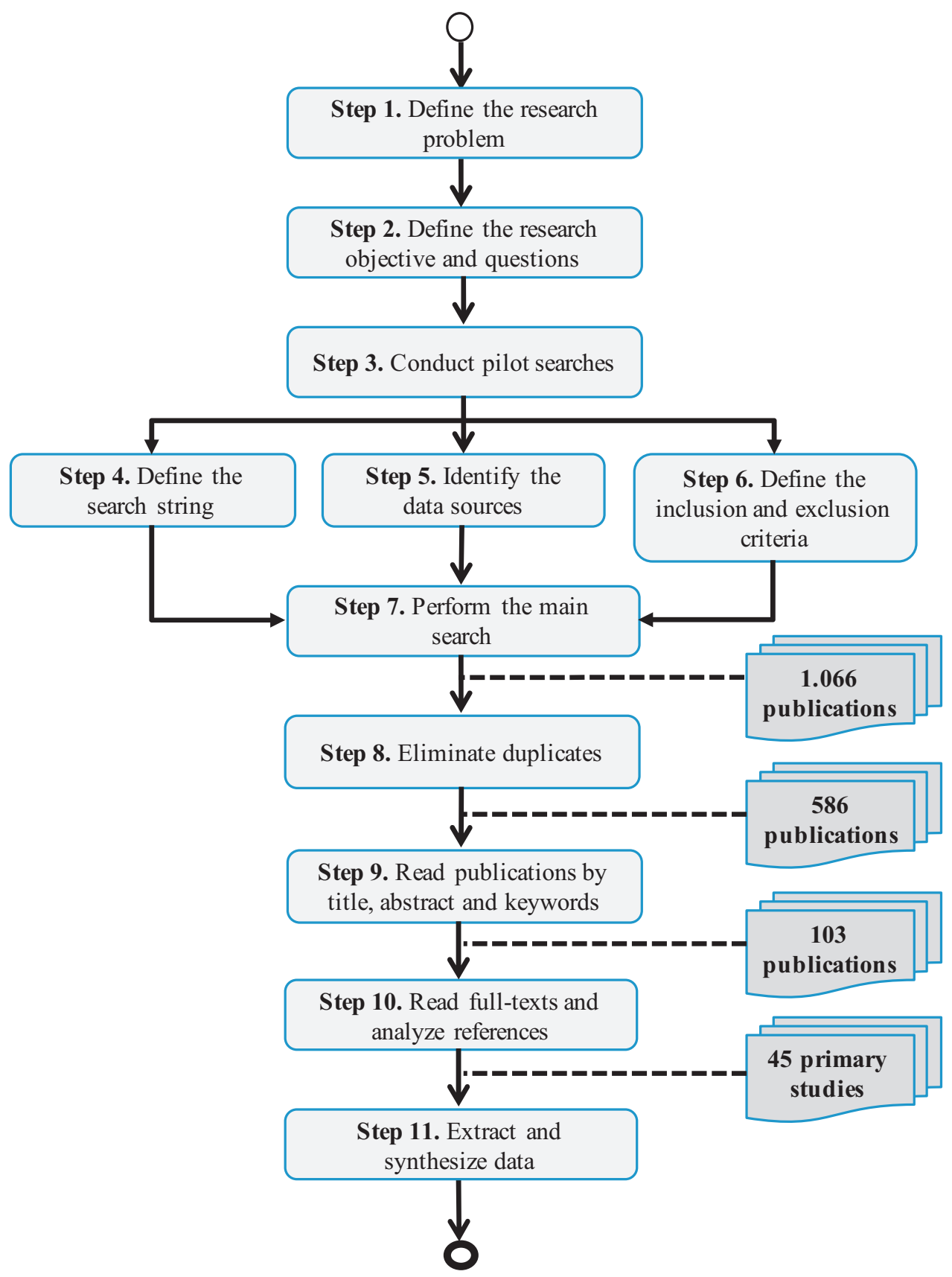

Fig. 1. SLR steps and resulting number of publications.

Step 4. Define the search string: The search string, which was formed according to our research questions, comprised of relevant keywords as refined in the example searches and preliminary literature review in Step 3. For the retrieval in the data sources (electronic libraries), the search string given below was derived and taken as the basis:

((understandability OR comprehension) and ("process model" OR $U M L)$ )

Step 5. Identify the data sources: In order to find the relevant studies, we searched the following six major electronic libraries: (1) Scopus (http://www.scopus.com), (2) ScienceDirect (http://www.sciencedirect.com), (3) ACM Digital Library (http://dl. acm.org), (4) Web of Science (http://apps.webofknowledge.com), (5) IEEE Xplore (http://ieeexplore.ieee.org), (6) SpringerLink (http: //link.springer.com).
Step 6. Define the inclusion and exclusion criteria: Based on our research objective and questions, we defined the inclusion and exclusion criteria to be applied on the resulting publications to identify those that are relevant to our research. Below are the inclusion and exclusion criteria we used for our review:

Inclusion criteria:

1. Publications published in English language.

2. Publications that are published between 1995 and 2015.

3. Publications that present empirical studies, which applied experimental methods involving participants (typically as process model readers), and analyzed the results using quantitative methods, such as statistical analysis methods.

4. Publications that focus on the factors that are considered to influence the understandability of visual 'process models' (modeled using graphical process modeling notations, such as Busi- 
ness Process Model and Notation - BPMN, Event-driven Process Chain - EPC) and of models that are depicted as Unified Modeling Language - UML behavioral diagrams. Such UML diagrams illustrate the behavior of a system and show very similar characteristics as process models. They are also commonly used for modeling processes [25]. These types of diagrams include [26]:

- UML Activity Diagram.

- UML Interaction Diagrams (Sequence Diagram, Communication Diagram, Interaction Overview Diagram, Timing Diagram).

- UML Use Case Diagram.

- UML State Machine Diagram.

By including the studies that investigate factors on the understandability of such models, we aimed at enriching the findings and strengthen our conclusions derived from the literature.

Exclusion criteria:

1. Publications in the grey literature; i.e. papers without bibliographic information (such as publication date/type, volume and issue numbers), working papers, or white papers.

2. Publications that investigate conceptual models [27] that are not in the form of business process/behavioral models, such as UML class diagrams and Entity-Relationship diagrams.

3. Publications which have enhanced, more complete and recent versions that offer a larger extent of contribution than the original paper.

4. Publications that do not investigate visual (graphical) process models.

Step 7. Perform the main search: As each electronic library provides slightly different search features, specific query strings and strategy were developed for each library taking the main search string formulated in Step 4 as the basis. As a general rule, the query strings were applied to the title, keywords, and abstracts of the publications residing in the libraries. (This is with the exception of Springer Link, which supports searching only full-texts and titles. Our search in this library resulted in 4993 publications, which were sorted by relevance. The first 167 publications were considered relevant, as further examination of publications between 167 and 250 did not identify any additional relevant work.) In total, 1066 publications were initially retrieved.

Step 8. Eliminate duplicates: Before applying the inclusion and exclusion criteria, duplicate entries resulting from the search of multiple electronic libraries were removed to generate a list of unique publications. After a careful review of 1066 publications, 480 were marked as duplicate, leading to 586 (unique) publications.

Step 9. Read publications by title, abstract and keywords: Each publication was reviewed based on the information provided in the title, abstract and keywords. Inclusion and exclusion criteria (except the 3rd exclusion criterion) were applied in this step for selecting relevant publications. As a result, 103 (out of 586) publications were identified for thorough investigation.

Step 10. Read full-texts and analyze references: We read full-texts of 103 publications. Re-applying the inclusion and exclusion criteria to these publications led to a refined list of 40 publications. We also analyzed the references of these publications and identified 5 additional studies following a snowballing approach proposed in [28] that were missing in our initial master list. This step led to a final list of 45 primary studies. Accepting a publication as a primary study meant that it would be used as a source to be used to answer the research questions of our SLR.

Step 11. Extract and synthesize data: For the thorough investigation, a data extraction form was constructed that defines the key data items to be collected for each publication. This involves information about the research method and design -including the investigated understandability factors and indicators used to measure model understandability, experimental setup, analysis method, and key findings. Data synthesis is performed to provide interpretive explanations.

For each understandability factor that has been investigated in a study, we analyzed the type of the influence (direct, moderation, or both) and its direction (no effect or significant effect) on the process model understandability indicators.

Type of the influence:

The influence of an understandability factor is considered to be direct when the change in the factor results a proportional change in the indicator used to measure understandability. For instance, the researchers have investigated if the structural complexity of a process model has a direct influence on its understandability.

In the case of moderation, the moderator factor influences the direction and/or strength of the relation between another (direct) factor and the understandability indicator. For instance, in the example given above, the model reader's process modeling experience can be investigated as a factor that moderates the relationship between structural complexity and understandability in such a way that the effect of complexity on understandability would be stronger for inexperienced model readers. In a study, a factor can also be investigated both for its direct and moderating influence.

Direction of the influence:

A factor is considered to have 'no effect' if the analysis shows no statistically significant influence of the factor on a process model understandability indicator (or in the case of moderation, on the relation between a direct factor and the understandability indicator).

In the case of a statistically 'significant effect', the direction can be of positive, negative or mixed type. The positive effect indicates a positive relation between the factor and the understandability indicator (i.e., an increase in the direct factor leads to an increase in the understandability). For instance, we can investigate if an increase in model reader's theoretical process modeling knowledge would lead to an increase in his/her model understanding.

The negative effect indicates an opposite direction. For instance, many researchers have investigated if (and under what conditions) an increase in the size of the process model leads to a decrease in its understandability.

The mixed influence is applicable typically for factors with nominal scales. For instance, researchers have performed studies comparing different modeling notations in terms of their understandability. Accordingly, some modeling notations can perform significantly better than others (that have been compared against) in facilitating understandability. Therefore, the direction of the influence for the notation factor can be positive or negative (mixed) depending on the particular modeling notation that has been chosen with respect to a set of other notations.

\section{General findings}

This section provides an overview of the general findings obtained from the analysis of the primary studies, focusing on the year and type of publications, and the setup of the experiments, as well as the process modeling notations used in the experiments.

The research in the area of understandability of process models mostly benefits from controlled experiments to find out how understandability is affected by different factors. Fig. 2 shows the distribution of primary studies by year (from 1995 to 2015). The number of publications has reached the highest number (12) at 2013. We observe that there is a general trend for increasing number of publications in the last recent years.

Out of 45 primary studies, 24 are published in journals, 15 in conference proceedings, and 6 as workshop papers. Fig. 3 presents the distribution of primary studies by publication type. By applying 


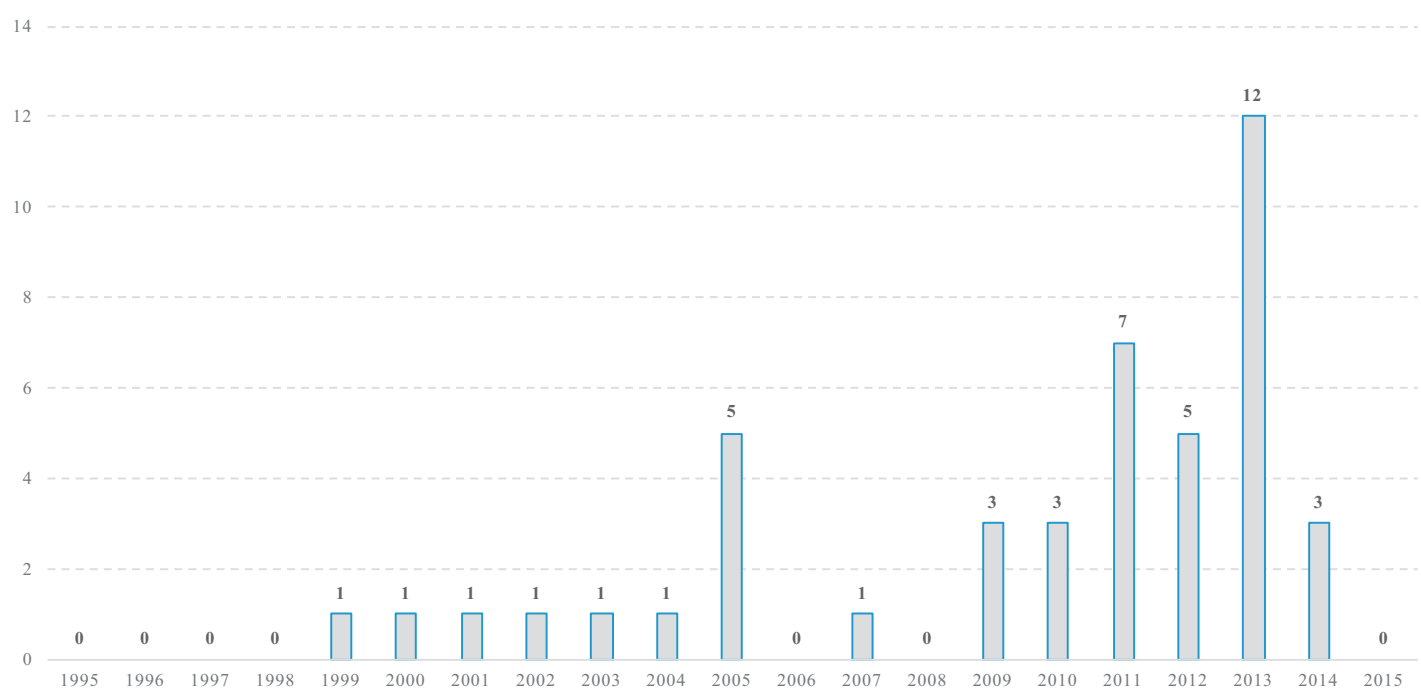

Fig. 2. Distribution of primary studies by year.

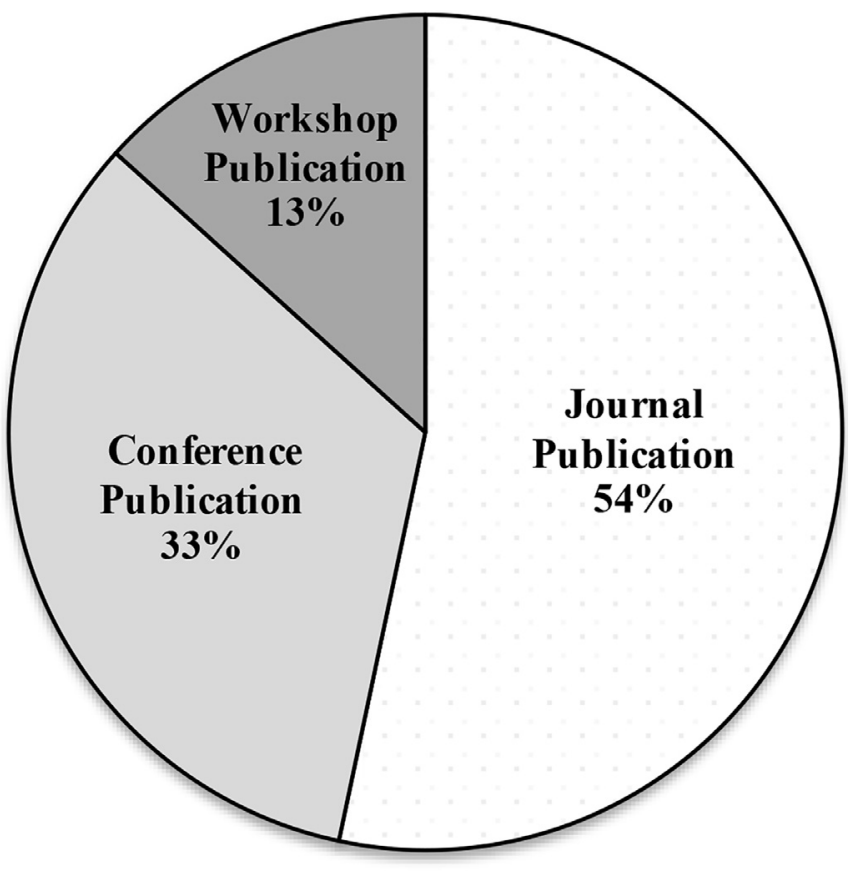

Fig. 3. Distribution of primary studies by publication type.

our 3rd exclusion criterion, 19 conference/workshop publications were taken out of the primary list, as these have recent versions with enhanced contributions that are typically published as journal publications. The results about the numbers and types indicate that the business process model understandability is a mature field and it is worth performing an SLR on this topic.

Four publications appeared in the Information and Software Technology journal, which corresponds to the highest number among the journals. The numbers for the conference publications indicate an even spread over the conferences. Only two conferences have two publications each (CAiSE - International Conference on Advanced Information Systems Engineering, and VL/HCC - IEEE Symposium on Visual Languages and Human-Centric Computing).

Fig. 4 shows the number of experiment participants used in each empirical study, including the ratio of students to industry practitioners. Accordingly, a large majority of the subjects (88\%) that participated in the experiments were students (bachelor, master or $\mathrm{PhD}$ ), whereas only $12 \%$ were industry professionals. The data for the empirical analysis originates from the participation of around 85 subjects on the average for each work.

The list of process modeling notations used for the experiments, the primary studies that use the notation and the number of primary studies that used the related notation are given in Table 1 . In the literature, 20 different notations were used in 41 primary studies that explicitly indicated the type of the process modeling notation that has been used. Some studies used more than one modeling notation, particularly for comparative analysis. BPMN and EPC are the most common notations in the empirical works in the process model understandability research. We should emphasize that studies listed in Table 1 that use these languages do not necessarily investigate their influence on the understandability, for instance by comparisons between languages. Some of these works use a single notation for a process model to investigate the effect of other factors.

\section{Process model understandability indicators}

In this section, we present the indicators that the studies in the literature used to operationalize process model understandability. In the conceptual modeling domain, the effectiveness and efficiency are typical indicators used to measure the understandability of a model $[29,30]$. Our study also found these as the most commonly used indicators in the process model understandability research field. In addition, we found four indicators that are used for quantifying process model understandability. Accordingly, we can categorize these indicators into two groups: objectively measured understandability and perceived understandability. While former aims at applying approaches to objectively measure model understandability (as described below in this section), the later relies on participants' perceptions on the level of model understandability. Table 2 presents these indicators including the studies that used them.

A brief description of each process model understandability indicator is given below.

PMUI 1. Understandability task effectiveness

In the studies that use this indicator, the participants of the experiments are confronted with a set of understandability tasks or questions about the process models that are used as the objects in the experiments. The understandability task effectiveness is computed as the number of correct answers (or successfully completed 


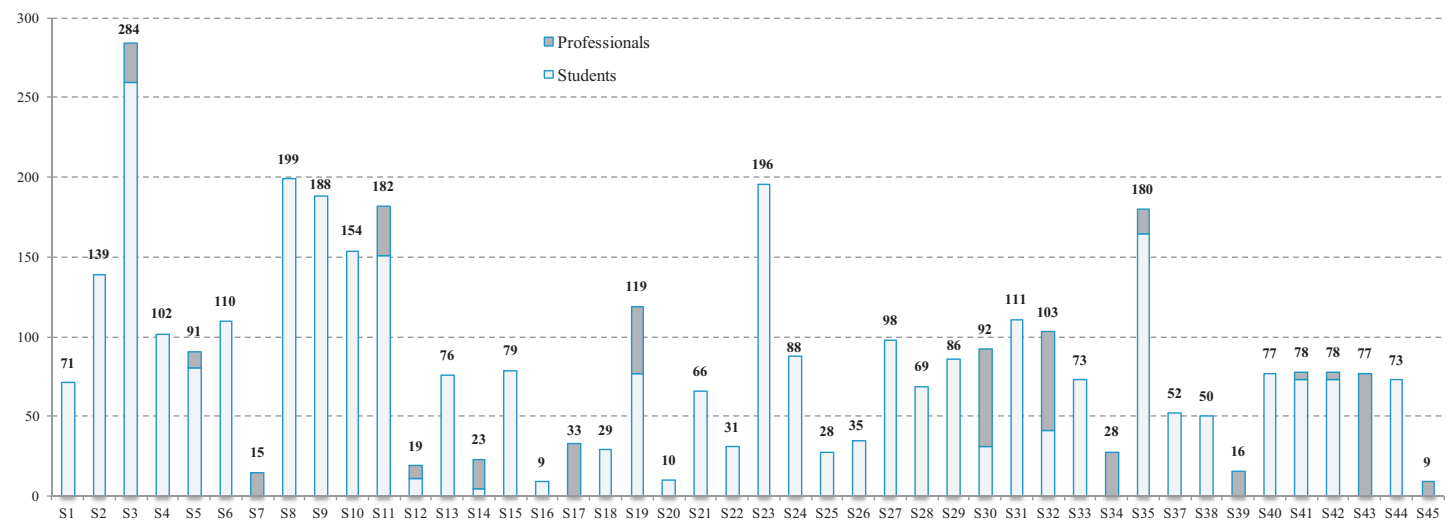

Fig. 4. Number of participants in experiments and distribution of participants (the number of experiment participants is not provided in [S36]).

Table 1

Process modeling notations used in primary studies.

\begin{tabular}{|c|c|c|c|}
\hline No & Process modeling notation & Number of primary studies & Primary studies \\
\hline 1 & BPMN & 15 & [S6], [S8], [S9], [S10], [S20], [S23], [S25], [S27], [S28], [S29], [S35], [S36], [S37], [S39], [S43] \\
\hline 2 & EPC & 11 & [S8], [S9], [S10], [S14], [S18], [S19], [S28], [S29], [S33], [S38], [S43] \\
\hline 3 & UML Statechart Diagram & 8 & [S3], [S4], [S5], [S12], [S21], [S22], [S41], [S42] \\
\hline 4 & UML Activity Diagram & 7 & [S8], [S9], [S10], [S14], [S31], [S40], [S43] \\
\hline 5 & UML Sequence Diagram & 6 & [S1], [S13], [S21], [S22], [S41], [S42] \\
\hline 6 & UML Use Case Diagram & 4 & {$[\mathrm{~S} 2],[\mathrm{S} 15],[\mathrm{S} 23],[\mathrm{S} 40]$} \\
\hline 7 & UML Collaboration Diagram & 4 & [S13], [S21], [S22], [S26] \\
\hline 8 & Petri Net / Workflow Net & 3 & [S32], [S34], [S38] \\
\hline 9 & Declare & 3 & [S16], [S25], [S45] \\
\hline 10 & YAWL & 2 & [S9], [S10] \\
\hline 11 & Health Process Notation (HPN) & 1 & [S39] \\
\hline 12 & Tropos & 1 & [S15] \\
\hline 13 & Restricted Use Case Modeling & 1 & [S44] \\
\hline 14 & Textual Representation & 1 & [S16] \\
\hline 15 & SBD (Storyboard) & 1 & [S43] \\
\hline 16 & SPEM & 1 & [S11] \\
\hline 17 & Data Flow Diagram & 1 & [S1] \\
\hline 18 & OML-Internal Collaboration & 1 & [S21] \\
\hline 19 & OML-State Transition & 1 & [S21] \\
\hline 20 & OML-White Box Sequence & 1 & [S21] \\
\hline
\end{tabular}

tasks) divided by the total number of questions in an understandability test [20]. As seen in Table 2, it is the most widely used indicator to evaluate the understanding of a process model.

PMUI 2. Understandability task efficiency

The majority of the studies in the literature that use this indicator in their work operationalize it by dividing the number of correctly answered understandability questions by the time it takes to complete the questions. In few studies ([S11], [S12], [S13]) it is operationalized only by the time it takes to complete the understandability test, without taking into consideration the number of correct answers during this time period.

PMUI 3. Cognitive load (mental effort)

According to Cognitive Load Theory, working memory can deal with only a limited number of elements at a given point of time [31]. When the amount of information to be processed exceeds this capacity, understandability is negatively affected. In other words, cognitive load should be as low as possible for higher understandability. Zugal et al. [32] proposed the mental effort indicator in addition to the understandability task effectiveness and efficiency to provide further insight into the understandability of process models. Mental effort corresponds to the mental resources required to solve a problem and is assessed by the user's rating as a subjective measure (perceived difficulty). In [S45], the subjects were asked to assess mental effort (cognitive load) expended in a 7-point rating scale after answering an understandability question [S45]. Cognitive load was considered as an indicator to operationalize the level of understanding in three primary studies [S8], [S16], [S45] and it is the least used indicator to operationalize process model understandability among six indicators.

The remaining three indicators are based on the primary constructs of the Technology Acceptance Model (TAM) that describes how users accept and use technology [33]. In the model understandability context, these indicators aim to capture model readers' perception on model's usefulness and ease of understanding, as well as their intension to use the model. TAM and its derivatives, such as [34], are commonly referred theories that predict and explain the acceptance and use of design artefacts, such as IS methods and models $[35,36]$. In TAM, the two constructs -perceived usefulness and ease of use, are believed to be strong determinants of users' intentions to use a design artefact. In the empirical works that we analyzed, the variables that were adopted are operationalized using multiple scale items, which have been evaluated for reliability and validity in previous research $[33,35]$. The typical operationalization of these indicators involves a set of arguments (scale items) with options to be selected in the form of Likert scales.

PMUI 4. Perceived ease of use (for understanding)

According to the TAM model, perceived ease of use indicates "the degree to which a person believes that using a particular system would be free of effort" [35]. In our context, 'a particular system' maps to a process model in a specific form, and 'effort' represents the mental effort, as in Houy et al. [20]. Typical quantification of perceived ease of use involves a set of questions with answers in Likert scale that aims to capture participants' subjective perception on the ease of use. 
Table 2

Process model understandability indicators and related primary studies.

\begin{tabular}{|c|c|c|c|}
\hline No & $\begin{array}{l}\text { Process model } \\
\text { understandability indicator }\end{array}$ & Number of primary studies & Primary studies \\
\hline \multicolumn{4}{|c|}{ Objectively measured understandability } \\
\hline PMUI 1 & $\begin{array}{l}\text { Understandability task } \\
\text { effectiveness }\end{array}$ & 38 & $\begin{array}{l}\text { [S1], [S2], [S3], [S4], [S6], [S8], } \\
\text { [S9], [S10], [S13], [S14], [S15], } \\
{[\mathrm{S} 17],[\mathrm{S} 19],[\mathrm{S} 20],[\mathrm{S} 21],[\mathrm{S} 22],} \\
{[\mathrm{S} 23],[\mathrm{S} 24],[\mathrm{S} 25],[\mathrm{S} 26],[\mathrm{S} 27],} \\
{[\mathrm{S} 28],[\mathrm{S} 29],[\mathrm{S} 30],[\mathrm{S} 31],[\mathrm{S} 32],} \\
{[\mathrm{S} 33],[\mathrm{S} 34],[\mathrm{S} 35],[\mathrm{S} 36],[\mathrm{S} 38],} \\
{[\mathrm{S} 39],[\mathrm{S} 40],[\mathrm{S} 41],[\mathrm{S} 42],[\mathrm{S} 43],} \\
\text { [S44], [S45] }\end{array}$ \\
\hline PMUI 2 & $\begin{array}{l}\text { Understandability task } \\
\text { efficiency }\end{array}$ & 25 & $\begin{array}{l}\text { [S4], [S5], [S9], [S10], [S11], } \\
{[\mathrm{S} 12],[\mathrm{S} 13],[\mathrm{S} 15],[\mathrm{S} 16],[\mathrm{S} 19],} \\
{[\mathrm{S} 21],[\mathrm{S} 22],[\mathrm{S} 25],[\mathrm{S} 26],[\mathrm{S} 27],} \\
{[\mathrm{S} 28],[\mathrm{S} 29],[\mathrm{S} 31],[\mathrm{S} 32],[\mathrm{S} 35],} \\
{[\mathrm{S} 36],[\mathrm{S} 37],[\mathrm{S} 39],[\mathrm{S} 40],[\mathrm{S} 41]}\end{array}$ \\
\hline \multicolumn{4}{|c|}{ Perceived understandability } \\
\hline PMUI 3 & Cognitive load (mental effort) & 3 & [S8], [S16], [S45] \\
\hline PMUI 4 & Perceived ease of use & 10 & $\begin{array}{l}\text { [S7], [S9], [S10], [S13], [S17], } \\
\text { [S18], [S20], [S28], [S38], [S39] }\end{array}$ \\
\hline PMUI 5 & Perceived usefulness & 5 & [S2], [S7], [S17], [S20], [S39] \\
\hline PMUI 6 & Intention to use & 7 & $\begin{array}{l}\text { [S7], [S17], [S20], [S26], [S38], } \\
{[\mathrm{S} 40],[\mathrm{S} 41]}\end{array}$ \\
\hline
\end{tabular}

\section{PMUI 5. Perceived usefulness}

Perceived usefulness represents “a person's subjective probability that using a particular system would enhance his or her job performance" [35]. In the model understandability context, it indicates users' perception on the utility of a process model in providing gains to the user in terms of understandability.

PMUI 6. Intention to use

Intention to use is defined as "the extent to which a person intends to use a particular system" [35]. The studies that use this indicator ask model readers to indicate their level of intention to use the proposed process model.

\section{Process model understandability factors}

In this section, we provide a detailed view on the factors that have been investigated in the literature. We propose Fig. 5 as an 'integrated framework of process model understandability' that shows all factors that have been investigated in the literature for their influence on model understandability. The framework also incorporates the indicators used for quantifying model understandability (as discussed in Section 4). Any empirical research that we analyzed in this study investigates the influence of at least one of these factors on at least one of the process model understandability indicator. Accordingly, we can distinguish two main categories of understandability factors: process model factors and personal factors. Later in this section, we describe these categories and related factors in detail.

In total, 20 factors (12 process model factors and 8 personal factors) were investigated in 45 primary studies. These factors with the number of primary studies in which that factor was investigated are presented in Fig. 6. The figure shows that, excepting the factor PF 8-Domain Familiarity, all investigated factors are found to influence process model understandability significantly (as there exists at least one primary study where a significant impact of that factor on at least one of the process model understandability indicators exists). There is a single study (S18) that studied the moderation effect of the factor of domain familiarity (PF 8), where the effect was found insignificant.

Table 3 presents the factors that were investigated in each study, including the type of the effect (direct, moderator or both) and whether the effect was found significant or not. The table uses the following convention to indicate this information:
- The sign ' $\sqrt{ }$ ' denotes a direct factor that has a significant effect (positive, negative, or mixed) on at least one of the process model understandability indicators.

- The sign ' $\mathrm{O}$ ' denotes a direct factor that has no significant effect on any process model understandability indicator.

- An additional '*' symbol after ' $\sqrt{ }$ ' or ' $O$ ' denotes that the factor is investigated as a moderator.

- An additional '+' symbol after ' $\sqrt{ }$ ' or ' $O$ ' denotes that the factor is investigated both for its direct and moderating effect.

Next, we elaborate on each understandability factor as studied in the literature.

\subsection{Process model factors}

\section{PMF 1. Modeling notation}

As we presented in Table 1, several process modeling notations were used in the studies that investigated the influence of various factors on process model understandability. Sixteen studies have examined the process modeling notation as an influential factor. A typical research design for these studies involved a comparative analysis between different modeling notations to investigate if a particular model outperforms the others with respect to model understandability. In 12 studies, using different notations resulted significantly different levels of understandability, which suggests that the process modeling notation can be an influential understandability factor. However, there are also conflicting results even in the experiments that use the same set of process modeling notations. In the paragraphs that follow, we briefly summarize the findings of these empirical works.

The study presented in [S43] compares the EPC, BPMN, and UML activity diagrams with respect to their influence on model understandability. The participants of the experiment reached lower scores in EPC models compared to models in UML and BPMN. However, some studies, such as [S29], argue that the impact of using EPCs or BPMN notation in terms of model understandability is insignificant. In a test presented in [S28], where participants had knowledge of only EPC notation, process model comprehension was measured for BPMN and EPC models, and no significant difference was observed for understandability task effectiveness, efficiency, and perceived ease of use. The study concludes with an argument that if the users of process models are comfortable with either of the modeling notations adopted by their organizations, 


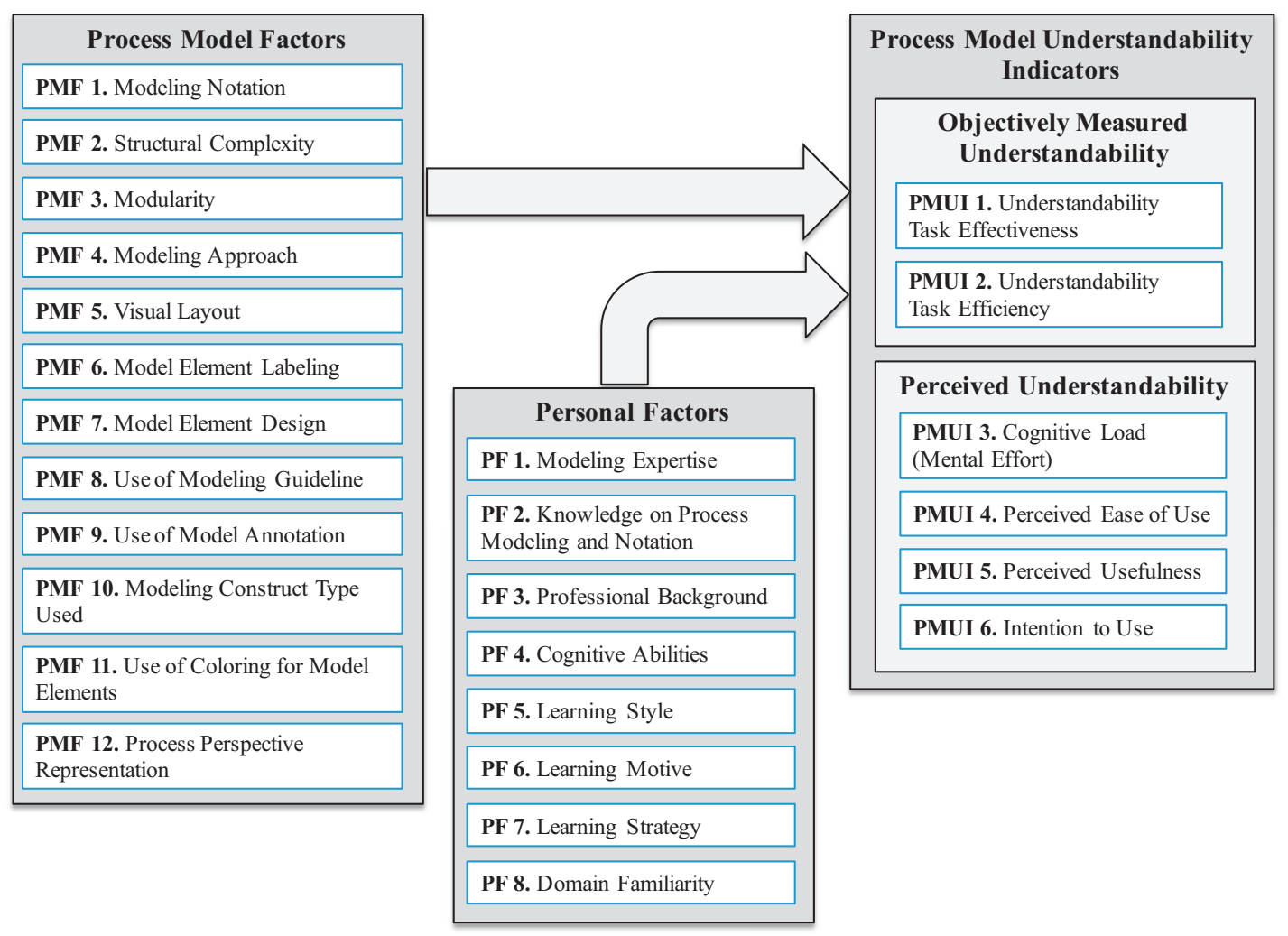

Fig. 5. An integrated framework of process model understandability.

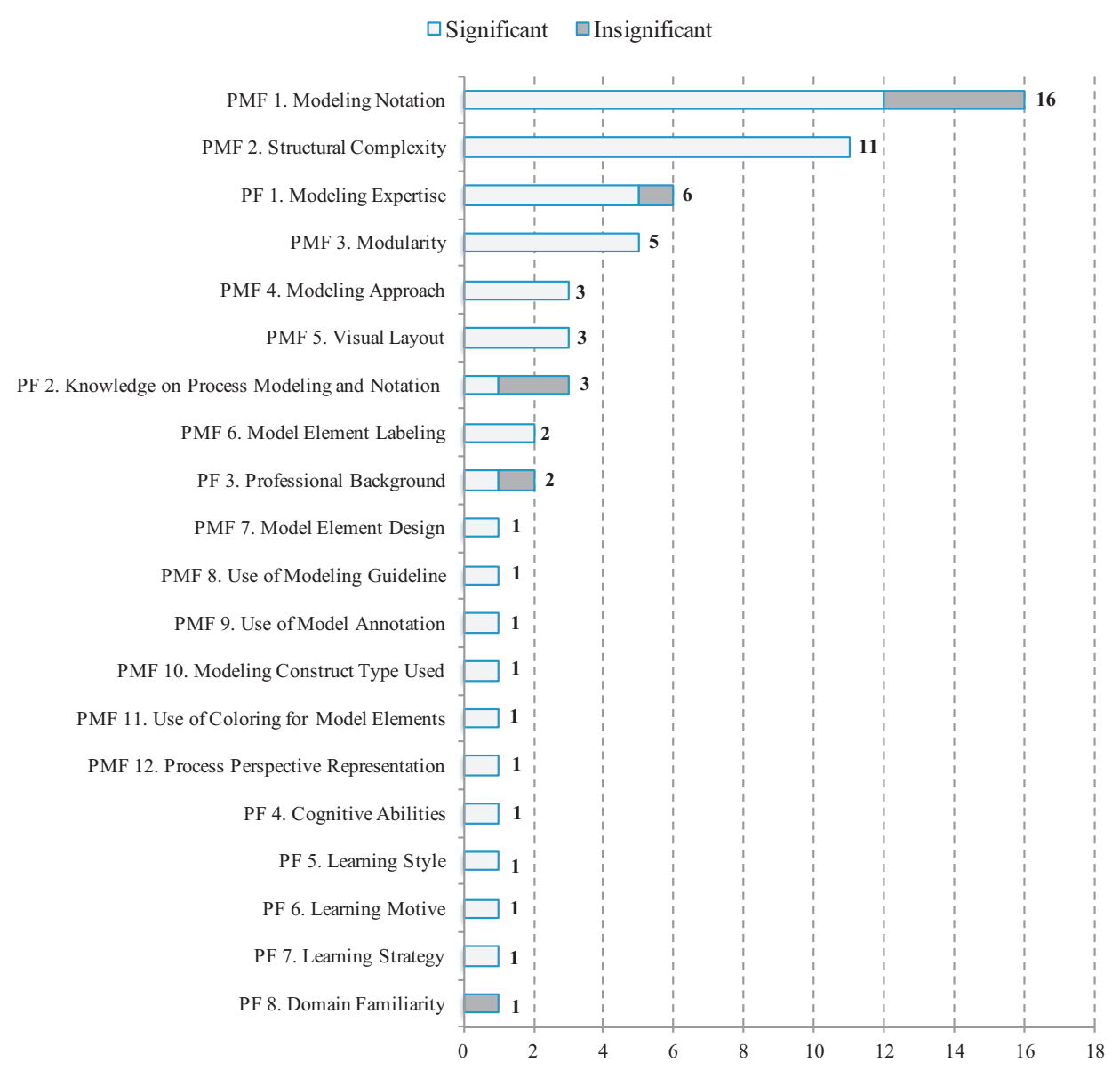

Fig. 6. Factors and number of studies that investigate those factors. 
Table 3

Factors investigated in primary studies.

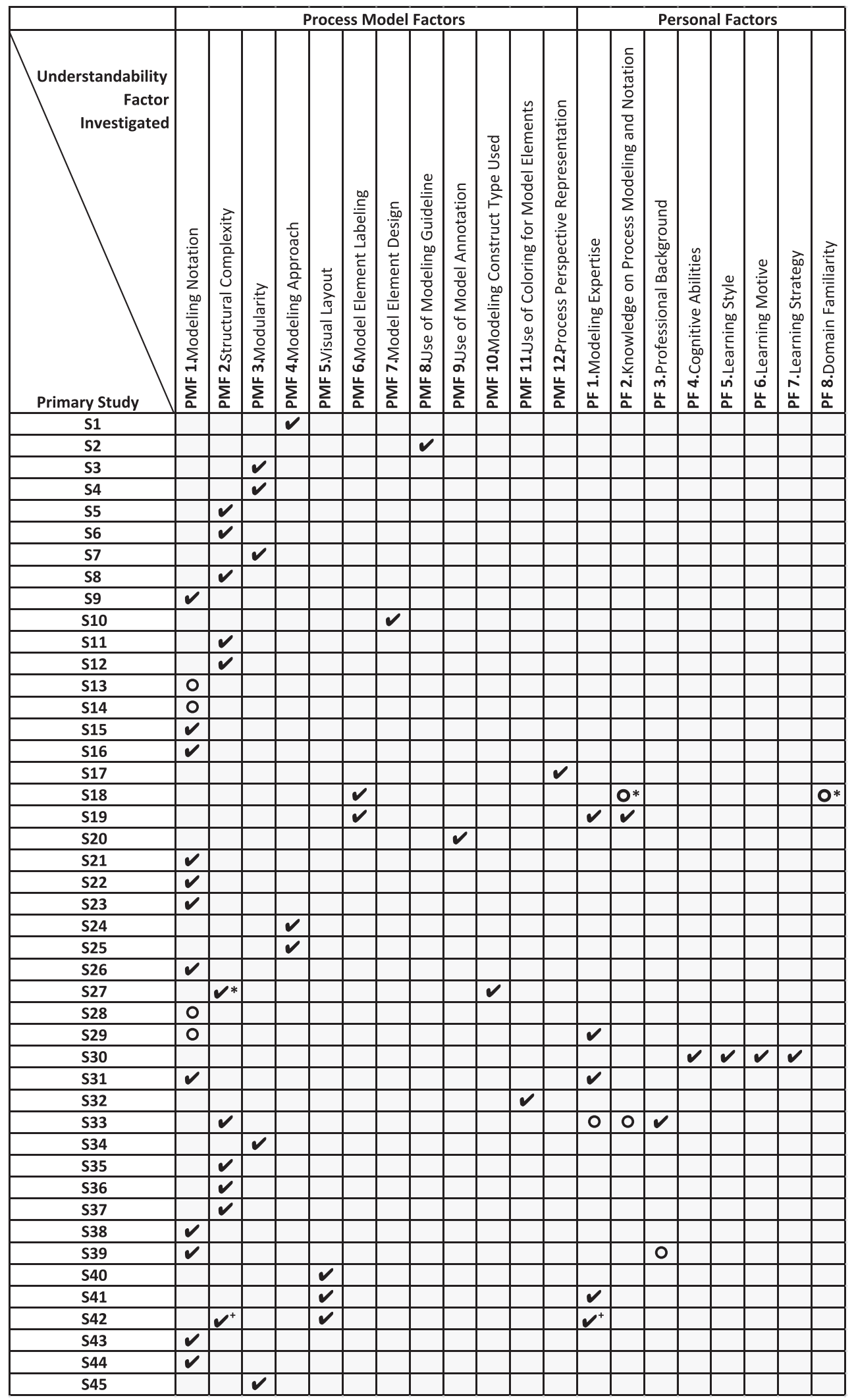


there is no need to switch to another notation [S28]. Similarly, the results of the experiment in [S14] with 23 participants, indicated no significant difference between UML Activity Diagrams and EPC diagrams in terms of their understandability.

The study presented in [S38] compares EPCs and Petri-nets, focusing on their approaches in representing the control-flow aspect of processes. The experiment involved 50 students with business and economy background. The process model understandability was quantified using model comprehension (effectiveness), perceived ease of use and intention to use. Significant differences were found in all three indicators favoring the EPC notation. However, the study discusses the use of a particular group of students in the experiment as a limitation, as students' motivation and learning style might not represent the population of the endusers in practice.

In an experiment conducted in [S9], four different symbol sets derived from the EPC, UML Activity Diagrams, YAWL and BPMN were compared. The results showed that the notational deficiencies have a significant effect on process model understandability.

There are also works that investigated the understandability of behavioral diagrams in UML. The study presented in [S13] examines the understandability of UML sequence and collaboration diagrams. The study concludes that there is no statistically significant difference of using any of these diagrams when their understandability is of concern. Similarly, the study presented in [S22] compares three UML diagram types that are used to represent the dynamic behavior, UML sequence, collaboration, and state diagrams. No significant difference is found between these notations in terms of the way they influence model understandability. In [S26], five independent notational variations differences in the notation used for UML collaboration diagrams are compared. Each notational difference has two variations with identical semantics. The results indicate a significant effect of notational variations on the understandability task effectiveness, efficiency and intention to use.

In [S31], the authors present the results of a family of controlled experiments which was conducted to evaluate the level of formality in workflow modeling. For this purpose, two styles that represent different levels of formality were used: a precise style and an ultra-light style for the UML activity diagrams. Analyses showed that the precise style yields significantly better understandability task effectiveness results, but the style used for modeling has no significant impact the on the understandability task efficiency.

The study presented in [S21] examines the semantic comprehension of UML and OML (OPEN Modeling Language) from the perspective of dynamic modeling - in particular, based on the interaction and the state diagrams of these two language families. The findings of the experiment with 64 students indicate that, for both model types, the specification of the dynamic behavior using OML is faster and easier to understand (understandability as task efficiency and effectiveness, respectively) than using the UML language. This is attributed mainly to the availability of logic boxes in OML to handle branching, looping and exceptions.

Other types of process modeling notations were also subjects to empirical works on model understandability. In [S15], a goalbased modeling language Tropos was compared with a scenariobased modeling language, i.e. the UML use case diagram. The understandability questions involved 14 questions that were asked to 79 subjects in three runs of an experiment to test participants' comprehension level and effort. Tropos models were found more understandable than use case models as measured by the understandability task effectiveness. However, the difference was not significant for understandability task efficiency. In [S16], the understandability of graphical and textual declarative process models is compared. The results indicate that the graphical representations are easier to understand. In a similar study, presented in [S23], authors examined the strengths and weaknesses of graphical and textual notations and compared the relative understandability of four alternative representations. The results indicate that the understandability is achieved best when the graphical models are complimented with the textual descriptions of a process (and vice versa).

\section{PMF 2. Structural complexity}

Increasing complexity in process models has been considered to impede the understandability and increase the likelihood of errors and defects in the models [37]. A research conducted on nearly 600 process models shows that larger process models tend to have more defects [38].

The literature suggests several measures to operationalize the structural complexity of process models. Our survey found the control-flow complexity (CFC) and size as the two most commonly referred measures in the literature. However, our review of empirical studies also revealed several other measures, such as the crossconnectivity and token splits [S33], structuredness [S6], [S33], diameter, density, depth and sequentiality [S33], [S36], connectivity and separability [S8], [S33], [S36], average connector degree, maximum connector degree, mismatch, and connector heterogeneity [S33], [S36], [S37]

Although some of the empirical works that we examined report conflicting findings, we observed an agreed convergence on the influencing role of model's structural complexity on its understandability. In the following paragraphs, we briefly summarize these findings discussing also the measures used in quantifying the structural complexity of process models.

The study presented in [S35] reports a significant correlation between the complexity as measured by the CFC measure and the understandability of BPMN models in terms of the understandability task effectiveness and efficiency. The CFC measure takes into consideration the number of decision points in the controlflow, i.e. XOR-split, OR-split and AND-split constructs in the BPMN models.

The studies presented in [S33], [S36], and [S37] use two measures as the representatives of the structural complexity of process models: maximum connector degree and connector heterogeneity. The maximum connector degree denotes the maximum sum of incoming and outgoing arc of the connector nodes in a process model. The connector heterogeneity denotes the extent to which different types of gateway constructs are used in a process model. The study presented in [S33] found no significant influence of complexity measured using these two measures on the understandability as measured using understandability task effectiveness. However, in [S36] and [S37] the complexity (as measured using the same measures) was found to influence the process understandability significantly (i.e. high maximum connector degree is correlated with decreased understandability, and lower variety of gateways used in a model is correlated with increased understandability). In [S36], the results also showed that the values of size and diameter have significant impact on the understandability task effectiveness. Similarly, the size, connectivity, diameter, density, average connector degree, mismatch, depth, and sequentiality have significant impacts on the understandability as measured by the understandability task efficiency.

The study in [S37] focuses on the gateway complexity due to the importance of gateways in the complexity of process models. The study involves a controlled experiment conducted to identify threshold values for a specific set of structural measures. Ten BPMN models were used, each with a different value of gateway complexity. The statistical analyses show that the control-flow complexity, gateway mismatch, average gateway degree, gateway heterogeneity, maximum gateway degree, and the total number of gateways influence the understandability (as measured by the understandability task efficiency). Moreover, threshold values for the 
structural measures were obtained to identify process models of good or poor quality in terms of process model understandability.

In [S8], two structural complexity measures are examined for their correlation with understandability: connectivity and separability. The connectivity between two elements is calculated as the number of arcs between two elements minus 1 (with the assumption that it equals to 1 if two elements are inside the same control block) and high separateness is accepted as existence of a cut-vertex between elements. The complexity, as measured using the connectivity measure, was found to influence understandability negatively (as measured by understandability task effectiveness and cognitive load). However, no significant correlation was found for separability.

The study presented in [S6] compares structured and unstructured process models in terms of their influence on process model understandability as measured by the understandability task effectiveness. Structuredness denotes the extent to which a process model is built by nesting blocks of matching split and join gateways. It requires that split gateways have always a matching join gateway and these pairs are nested within each other. Structuredness is a desired property as unstructured process models tend to have a higher probability of having errors [39]. The study found out that a structured model is more understandable, as long as structuring a process model does not increase the number of gateways more than the number of gateways in the unstructured version of that model [S6]. However, the study presented in [S33] reports conflicting findings which show no significant effect of structuredness on the understandability.

Studies presented in [S5], [S11], and [S12] examine the effect of structural complexity on the understandability of UML state diagrams. A set of measures, such as control-flow complexity and size are proposed to measure the structural complexity of the state diagrams. The results indicate no significant correlation between structural complexity and understandability. The study presented in [S27] investigates the structural complexity as a moderator factor on the relationship between the use of gateways and understandability task effectiveness and efficiency. The results indicate a significant moderation effect of the structural complexity on the mentioned relationship.

In [S42], the structural complexity is investigated both as a direct factor and a moderator factor that moderates the relationship between size and understandability. The diagram size (with two levels: small and large) was defined as the number of elements in a diagram, weighted by their complexity. The results indicate that the size influences the understandability task effectiveness negatively. The influence of layout quality on understandability task effectiveness was strengthened with increasing diagram size.

\section{PMF 3. Modularity}

Hierarchy through the use of sub-processes has widely been considered as a practical means to deal with the size and complexity of models [S45] and [40]. Many modeling languages allow for the design of hierarchical/modular structures, for example, subprocesses in BPMN and EPCs. Hiding less relevant information in sub-models is expected to decrease the mental effort (cognitive load) needed to understand the model [41], whereas fragmentation due to modularization increases the mental effort by forcing the reader to switch attention between different fragments (so called the split attention effect [S45]). The discussions about the proper way of using modularity and its implications on the understandability of models have not been conclusive [S7], [S34], [S45].

The works by Reijers et al. ([S34] and [40]) test the influence of using sub-processes on the understandability of two real-life processes that are modeled using Workflow Nets in two forms: modular and flattened. The participants (28 consultants) were asked to answer a set of (control-flow related) understandability questions regarding these models. For the first process model, the experi- ment did not result in a significant difference between the modular and flattened versions, but a positive influence of modularity on understandability was found for the second model. The authors attribute this to the difference in the degree of modularization applied in these models. As the second model had more sub-processes, they sparingly conclude that 'modularity appears to have a positive connection with process understanding'.

The study presented in [S45] tested the effect of modularization on the understandability of declarative process models. Four processes were modeled in two forms (modular and flattened) using a declarative language DecSerFlow [42]. The understandability is measured using the understandability task effectiveness, and the (perceived) mental effort. The results suggest that modularization decreases perceived mental effort but has no influence with respect to task effectiveness. The limited number of participants ( 9 respondents) is reported as a threat to the validity of the findings.

The study presented in [S7] used the expert evaluation approach (with 15 process modeling experts) to determine whether some visualization strategies provide a better fit for representing process model hierarchies than others. Accordingly, the experts preferred to navigate in the hierarchy with the help of an overview + detail strategy (where sub-processes are shown as separate models detached from the context of the higher-level model) instead of a focus + context strategy (where sub-processes are expanded in the higher-level model directly within their context). The 'overview + detail' view was observed to simplify the design and provide undistorted views on focus and context.

The study presented in [S3] presents a family of experiments investigating the effect of hierarchy on the understandability of UML state diagrams. The results indicate insignificant or varied effects of hierarchy on understandability. Moreover, the study [S4] reports a worsening understandability with the increase of the nesting level (depth of hierarchy).

This diversity in the results can be attributed to the outcome of two opposing effects of modularization: abstraction (information hiding) and split-attention effect (browsing costs) [43,44]. Using sub-processes might increase reader's understanding of a complex model by abstracting away less relevant information (and thereby reducing complexity). However, additional cost (increased cognitive load) incurred in browsing through and integrating fragmented pieces of models can counter-balance this gain [45]. It has been demonstrated that a user of a process model understands visual models by decomposing into smaller chunks which correspond to sub-processes and then connecting those chunks later $[41,46]$.

The impact of modularity on process model understandability has been examined using different process modeling languages, such as UML Statechart Diagrams [S3], [S4], Workflow Nets [S34], and DecSerFlow [S45]. In particular, there is a lack of studies on the effect of modularity that involve eEPC or BPMN, which are the de-facto process modeling notations in practice [47]. BPMN v2.0 has specific elements and techniques for representing modularity, such as collapsed/expanded sub-processes and groups, which have not been addressed in the research concerning process model understandability.

PMF 4. Modeling approach

The factor of modeling approach relates to a diverse set of dimensions regarding the methods used in modeling the processes. This typically includes the primary focus of attention or the main driver in modeling the processes. These approaches in process modeling include, for instance, the declarative vs. imperative modeling approaches [S25], object-oriented vs. process-oriented [S1], or artifact-centric [48] vs. activity-centric vs. role-centric [14] approaches. It is often the case that different approaches use (or sometimes require the use of) different process modeling notations. In that respect, such studies inherently test the understandability of process models represented by different modeling nota- 
tions (which are typically common and - in many cases - representative for the modeling approach). However, the primary focus and the definitive factor under investigation in these works are typically the modeling approach rather than the notation. The studies that we analyzed indicate a significant impact of the modeling approach on the understandability of process models [S1], [S24], [S25].

In [S1], semantically equivalent $\mathrm{OO}$ (object-oriented) and $\mathrm{PO}$ (process-oriented) models were used in an experiment and the understandability of models were compared in terms of the accuracy of understanding. The study found that a PO model is more understandable, but only for questions involving both structure and behavior.

The study presented in [S24] compares two methods; OPM (Object-Process Methodology) and OMT (Object Modeling Technique) in terms of understandability. A controlled experiment was conducted with 88 students, where the understandability was measured by the participants' responses to a questionnaire consisting of 33 questions. Statistical analyses showed significant differences between the two methods and a single model methodology. OPM was found to be more understandable than a multi-model methodology; OMT. Moreover, most of the participants preferred OPM to OMT.

Following a similar approach, the study presented in [S25] examines the understandability of the imperative and declarative process modeling approaches. The understandability had two dimensions in this empirical study, accuracy and speed. The statistical tests demonstrated that the imperative models are more understandable in terms of accuracy and speed than declarative models.

PMF 5. Visual layout

Several works stress on the importance of the visual layout of a process model as an influential factor for user's understanding of the process model $[49,50]$. Bernstein and Soffer [49] have identified a set of key visual layout features of process models, derived measures from these features, and applied them to sample process models.

There are also a number of empirical works, such as [S40], [S41] and [S42], that investigate the importance of this factor on model understandability. These three works derive their results from a series of experiments with 78 participants. The study [S40] uses UML use case and activity diagrams, where [S41] and [S42] use UML sequence and state diagrams as experimental materials. The quality of layout of process models used in the experiments was measured by the compliance or non-compliance to a number of layout rules, and had two levels, good layout and bad layout. The results indicate that the visual layout has a significant effect on the understandability task effectiveness, efficiency, and intention to use. The studies presented in [S41] and [S42], which use different types of diagrams confirm the findings, i.e., a good layout increases process model understandability.

PMF 6. Model element labeling

Individuals handle information better if it is provided through both auditory (i.e. words) and visual (i.e. images) channels. Accordingly, it can be expected that process model understanding can be improved when a better guidance for process model element labeling is provided. The study presented in [S18] found that verb-object style labels (for example, 'export license check') are regarded as more useful for understanding the process model than action-noun style labels (for example, 'notification printing') or rest style labels (for example, 'status analysis cash position') for activity labeling.

In [S19], the effects of the use of abstract versus concrete activity labels on the understandability task effectiveness (or performance) and understandability task efficiency are examined. Authors expected that the comprehension occurs quicker for people dealing with process models with abstract textual labels as they require less effort to retrieve and assemble pieces of information, when only having to consider graphical constructs but not additional textual information. It was found that both understandability task performance and understandability task efficiency were improved when activity labels were omitted.

PMF 7. Model element design

The process modeling notations support the expression of convergence and divergence semantics by using different visual symbols. The study presented in [S10] tests several hypotheses regarding the effects of perceptual discriminability, pop out, semantic transparency and aesthetic design of routing symbols on process model understandability. The findings indicate that the routing symbol design principles influence the understandability task effectiveness, but have no significant influence on the task efficiency.

PMF 8. Use of modeling guideline

The work in [S2] studied the effect of using modeling guidelines on model understandability. For this purpose, an experiment was conducted with 139 students. Three different sets of guidelines were used to construct and document use case models. In order to evaluate understandability, the participants answered questions about the functionality in the use case models developed by different guidelines. A significant difference in the understandability was observed if the use case models are constructed with different guidelines. Also, a significant difference in the usefulness of different guidelines was observed.

\section{PMF 9. Use of model annotation}

The study presented in [S20] proposes a context-based process semantic annotation model, where the authors test the effect of the annotation model on searching, navigation and understanding of process models. The results indicate no significant difference between the annotated and un-annotated process models in terms of understandability task effectiveness. However, most users perceived the annotated models as easy to use and useful for searching, navigating and understanding the process models.

PMF 10. Modeling construct type used

There have been only few works on the effect of using different generic process modeling constructs. The experiment conducted in [S27] with 98 students found that the understandability of process models decreases when the gateway constructs are not used. The experiment involved the use of three process models, where the authors used abstract activity names (such as task "A") in order to offset any potential impact of the domain knowledge.

PMF 11. Use of coloring for model elements

In [S32], the authors conducted an experiment to investigate the potential effects of syntactical element highlighting on the understandability of process models. The study argues that the use of colors to highlight matching operators has two advantages to improve understandability. First, it helps to identify a decomposition of the process model into components that enhances information hiding and, second, it helps to interpret secondary notation quickly since color can be processed by the humans much faster than graphical constructs. The experiment revealed that the highlighting of matching operators influences the understandability task effectiveness positively for novice process modelers. However, the influence is insignificant when the understandability task efficiency is concerned.

\section{$P M F$ 12. Process perspective representation}

The control-flow aspect of processes is often the primary and the only perspective represented in process models. However, in some cases it may be useful (or even required) to model data and resource perspectives of processes as separate models or incorporate them in models that show control-flow information. Visualizing the data objects and resources in the form of organizational units in process models with satisfactory readability and granularity on the available space poses challenges to the understandability of these models. 
In [S17], three visualization techniques - single view, multiple views, and multiple views in connection with linking and brushing, are investigated for their influence on process model understandability. Single-view shows all three perspectives blended on the same process model. In multiple-view, these perspectives are represented in different models (control-flow model, data model, resource model) and shown to the user at the same time. The third technique enriches the multiple-view with 'linking' and 'brushing'. The presentation is in such a way that if an item is selected or highlighted in one view (brushing), the corresponding connected items in the other views are also selected and highlighted (linking). Each participant in the experiment evaluated five process models, which were represented using a basic process modeling notation for each perspective. Four indicators were used to quantify understandability: understandability task effectiveness, perceived ease of use, perceived usefulness and intention to use. Statistical results showed that, although these models indicate no significant difference in terms of understandability task effectiveness, the visualization technique of showing multiple-views in connection with linking and brushing is preferred over single-view and multiple-views in terms of usefulness and ease of use.

\subsection{Personal factors}

The research confirms the significant impact of personal factors on process model understandability. Some researchers even argue that the personal factors have higher impact on process model understandability than the process model factors [4].

\section{PF 1. Modeling expertise}

Modeler's expertise is often considered as an important success factor of effective process modeling and critical for a successful BPM project [51]. A global survey with 529 BPMN users about the effect of modeling expertise on the perceived usefulness and perceived ease of use of a process modeling grammar reinforces the importance of this factor [52]. The study shows that the experienced process modelers can refer to their experiences for challenging modeling cases and interpreting complex process models. On the other hand, for the less experienced modelers, lack of this experience influences their effectiveness and efficiency of understanding in the opposite direction [53]. According to the resource allocation theory [54], the users that built up experience in modeling require less cognitive load in performing model-related tasks. Hence, some studies suggest to take different levels of expertise into account in providing guidance for process modelers [55].

Typical operationalization of the modeling expertise involves modeling experience and modeling intensity to distinguish between modelers that have modeled for a long time and those that model often [S19].

The study presented in [S29] tests the effect of modeling experience on understandability (as measured by task effectiveness and efficiency) by taking into account two aspects: transfer ability and retention ability. Transfer ability test measures deep understanding, while the retention ability test quantifies surface understanding. The experiment involved 68 postgraduates (in an information systems program), who were asked to indicate their modeling experience by estimating the number of process models that they have created or worked with. The participants were divided into two groups, above and below the median, corresponding to 36 and 32 participants. The results indicated that higher experience in process modeling resulted in significantly higher scores on the transfer ability, while it had no effect on retention ability scores (i.e. task effectiveness). With respect to task efficiency, the transfer and retention ability task completion times were not significantly different.

In [S31], modeling experience is considered as a factor that potentially influences the understandability of UML activity diagrams.
A significant effect of experience on the understandability (as measured using task effectiveness) was observed, indicating that the experienced people understand the workflows better. Similarly, the study presented in [S41] presents three experiments with 78 participants in total, where the effect of process modeling experience on the understandability of a set of UML diagrams was tested. The analyses showed that the task effectiveness increases with the increasing experience level. In [S42], the influence of modeling experience was investigated both for its direct and moderating influences. The results indicate that the expertise level has a direct and significant effect on the understandability. As for the moderating effect, the findings show that the subjects with higher modeling experience are much less affected by the increasing diagram size and poor layout than subjects with lower modeling experience.

The literature also reports on studies that were not able to confirm any relationship between modeling experience and understandability. The study presented in [S19] examines the effect of modeling experience and intensity on process model understandability. This work quantifies experience using four levels: less than one month, less than a year, less than three years, and longer than three years. The intensity also uses four levels to quantify the frequency of encountering process models in practice: daily, less than once a month, more than once a month, and never. The findings indicate no significant effect of the modeling experience and intensity on understandability task effectiveness, while the intensity influences the understandability task efficiency significantly. Likewise, in [S33], the modeling expertise is operationalized using the intensity of modeling, which is measured on a four-point scale ranging between "I never use business process modeling in practice" and "I use business process modeling in practice every day". The findings suggest no significant effect of modeling intensity on model understandability.

PF 2. Knowledge on process modeling and notation

This factor represents a person's theoretical knowledge on the general process modeling concepts and on the specific modeling notation used. A common way to measure this factor is to ask participants to self-assess their level of theoretical knowledge (as in [S33]). However, there are also works that use more reliable approaches and offer participants a short test to measure their level of theoretical knowledge on process modeling and notation (as in [S19]).

In [S33], the respondents self-assessed their theoretical knowledge on process modeling on a five-point ordinal scale, with anchor points "I have weak theoretical knowledge" and "I have strong theoretical knowledge." The findings indicate no significant influence of this factor on understandability task effectiveness. The study presented in [S18] follows a similar approach to quantify respondents' knowledge on a particular modeling notation. Based on self-assessments, the participants were put into two groups; high and low familiarity with the notation. This work investigates the knowledge of process modeling notation as a moderator factor on the relationship between labeling style and perceived usefulness. The analysis of 174 responses coming from 29 subjects indicate no significant effect of this factor on the relationship between label type and perceived usefulness.

On the other hand, the study presented in [S19], which used a test with 12 questions to measure respondent's level of theoretical knowledge, found a positive influence of knowledge on process modeling and notation on both understandability task effectiveness and efficiency.

\section{PF 3. Professional background}

In the model understandability research, the professional background covers a broad concept, which is typically used to represent participants' domain of work or education. In [S33], for instance, this factor is investigated as a categorical variable referring to the educational institute that the respondents are registered at. The 
results of the experiments indicated that students with different backgrounds score significantly different results in terms of model understandability. However, as the authors acknowledge that these students took different level of courses on process modeling, one can argue that this factor - as designed in this study, relates heavily on other personal factors - particularly to the factor of knowledge on process modeling and notation.

The study presented in [S39], however, operationalizes this factor using participants' professional background. The participants were referred to as those with an engineering background and others that have a background in healthcare. Yet, the results demonstrate that the professional background does not have a significant influence on the understandability task effectiveness, understandability test efficiency, perceived ease of use, or perceived usefulness. On the other hand, a significant interaction between the process model (BPMN or HPN modeling notation) and professional background was found out. In particular, engineers understood simple items better with the BPMN process model.

\section{PF 4. Cognitive abilities}

In [S30], a simulation experiment was conducted to test the effect of individual cognitive abilities on process model understandability. Cognitive abilities were operationalized by abstraction ability and selection ability. Abstraction ability allows an individual to constitute an abstract model for an entity of the external world [56], whereas selection ability enables an individual to search through a set of objects, attributes or relations in typically large diagrams with many informational artifacts [57]. Selection ability and abstraction ability were found to influence process model understandability in opposite directions, the first one in positive direction and latter one in negative direction.

\section{PF 5-7. Learning style, motive, and strategy}

Users can process information in several ways such as by seeing and hearing, reasoning logically or analyzing graphically in terms of learning style [58]. As users learn from a (graphical) process model, learning styles of users are considered as a factor in a number of studies [S30]. Felder and Silverman [58] examined the differences between sensing and intuitive learners. Sensing learners tend to memorize materials where intuitive learners are more comfortable with abstractions and prefer discovering possibilities and new relationships [59]. In [S30], a sensing learning style was compared with an intuitive learning style to test the effect of learning style on process model understandability. A sensing learning style was found to be more effective than an intuitive learning style in terms of understandability.

Learning motive indicates a person's desire in learning process and determines the person's perception of requirements of learning. Two types of learning motives can be identified, surface motive or deep motive [60]. A surface motive is triggered by extrinsic motivation such as aiming to meet expectations of superior. The study presented in [S30] examines the relationship between surface learning motive of a user and process model understanding performance. The results demonstrate that a surface learning motive negatively influences process model understandability.

In addition to learning style and motive, the study presented in [S30] investigates also the effects of the model reader's learning strategy on process model understandability. In this context, the learning strategy indicates the readers plan about how he/she will learn from a process model. A deep learning strategy aims at developing a thorough level of understanding for solving complex tasks and enables discovery of new knowledge. A surface learning strategy implies simple learning. The individual tries to memorize the content of the process model without questioning it. Surface learning strategy was found to increase process model understandability.

\section{PF 8. Domain familiarity}

In the software engineering field, the research confirms the effect of prior knowledge of the application domain on the understanding of software source code [61]. According to the Cognitive Theory of Multimedia Learning (CTML) [62], prior knowledge of the domain covered in a conceptual model lowers the cognitive load required to develop a mental model of the information represented in the model. As a result, it becomes easier to understand the model.

The study [S18] investigates the moderation effect of the domain familiarity on the relationship between labeling style and perceived usefulness. The respondents were categorized into two groups based on their self-assessment: those that have high application domain knowledge and those that have low. However, the findings of the analysis showed no significant moderation effect on the relationship between labeling style and perceived usefulness.

\section{Discussions}

In this study, we reported a systematic literature review on the factors that have been investigated in the literature and that are considered to influence the understandability of process models. In doing so, we also reviewed how process model understandability has been operationalized in the literature. We performed searches on the established electronic libraries for potentially relevant studies published between 1995 and 2015. A total of 1066 publications were identified following the searches in 6 electronic libraries. We selected 45 primary studies based on inclusion and exclusion criteria. Based on our two research questions; we extracted data and empirical evidence from the studies and synthesized them to answer our research questions.

Our first research question involves the way the process model understandability has been operationalized in the literature. We investigated 6 indicators that the researchers used to measure the understandability of process models. As presented in Table 2, the understandability task effectiveness is the dominant indicator used to quantify understandability. The typical setup to compute this indicator involves a set of understandability questions related to the process models used in the experiments to be answered by the participants. Tracking the time that participants spend in answering these questions forms the basis for another commonly used indicator - the understandability task efficiency. The set of indicators used for process model understandability also involves subjective measures, such as the perceived ease of use, that are based on technology acceptance models. These indicators aim to capture participants' perception on the level of ease of use and usefulness of the process models for understandability.

Looking closely on the studies that use the task effectiveness to quantify understandability, we see very few of them reporting on the questions that the participants of the experiments were asked or the methodological steps and concerns that were taken into consideration in developing such questions. This is important, as the reliability of this indicator - and thus the construct validity of the experiment, depends heavily on the quality of the understandability questions. The number of questions used for the experiment, their representativeness and appropriateness for the particular research hypotheses, and their level of difficulty are some factors that can potentially yield unreliable and inconsistent results - even within the same experimental settings. For instance, the findings of the empirical study in Laue and Gadatsch [63] indicate that the scores that the respondents got from the understandability questions are influenced significantly by how understandable these questions are to their audience. Empirical studies in this field should follow and clearly present a rigorous approach in conceptualizing and operationalizing the term process model understandability to ensure valid findings. 
Our review of literature on this specific topic can act as a reference to bring about a more consistent understanding of the concepts and use of terminology in process model understandability. Practical guidelines on operationalizing understandability would help researchers in designing, conducting, and reporting of sound and valid experiments. This can also allow comparing results of different empirical studies. A standard way of reporting on an experiment would provide valuable information on the review of articles, replication of the experiments, and analysis, comparison and interpretation of the findings [64].

To answer our second research question, we had a detailed look into the factors that have been investigated in the literature using empirical methods. Apart from the general findings on the type and the composition of the participants in each work, we derived a list of factors that have been tested for their influence on process model understandability. We thoroughly analyzed the studies to identify these factors. We looked how a factor was operationalized, how its effects were tested, whether it was influential to model understandability, and if so how. Reviewing 45 primary studies, we identified 20 factors investigated in the literature. These factors and the understandability indicators are integrated into a framework of process model understandability and depicted in Fig. 5.

The results of our empirical study give insight on the body of research on the factors of process model understandability. The studies that compare process modeling notations in terms of their influence on the understandability (corresponding to the studies referred to in PMF 1 in Section 5.1) form the majority of the empirical works in this field. The next commonly investigated factor is the structural complexity of the model, which has been measured using various measures discussed in Section 5.1 under PMF 2. There is a shared consensus in the community about the negative influence of the structural complexity on understandability. However, the comparative studies among process modeling notations are far from agreeing on the notations that are more understandable over the others. They also do not provide a clear insight on the characteristics of process modeling notations that contribute or deteriorate understandability.

In addition, majority of the studies that compare notations or investigate the influence of structural complexity focus only on the control flow aspect of processes. Typical objects of the experiments in these studies are one or two process models only with control flow information. This is limited, as the resource (organizational) and data (information) perspectives of processes are useful and highly relevant, particularly when the main purpose of process modeling is to communicate process information. However, to help achieve that purpose, we need to understand how and under what conditions the understandability of process models is influenced as these perspectives are incorporated into models [65]. On the other hand, incorporating more information in models increases its size, which is also known to hinder understandability [66].

Looking further at the influential factors, we observe that the process model factors have been intensively investigated, while the personal factors have received less attention. Very few studies have investigated the combined influence of process model factors and personal factors and their relationships. Majority of the experiments reported in the literature use process models of different size and complexity, and with participants of different background such as student and practitioner, process modeling experience, and knowledge. Contradictory findings in these studies signify how complex the interrelationships between these factors are. There is a need for comprehensive empirical works that uncover the relationships between influential factors to understand the context under which such factors become effective in improving or hindering model understandability. For instance, we know little about the thresholds for various measures of process model size and complexity over which the understandability of a process model starts deteriorating for a particular audience with certain process modeling expertise and knowledge. Lack of such studies prevent us in synthesizing clear and practical guidelines for creating understandable process models. There are only very few works (such as the Guidelines of Business Process Modeling [13], and Seven Process Modeling Guidelines - 7PMG, for structuring models [21]) that provide suggestions for better process models and that rely on sound empirical evidence. Lack of such works also makes it difficult to form a basis for future empirical works in the field.

Our analysis showed that using students in the experiments is the dominant approach in the field. Majority of the studies used student populations ( $88 \%$ of all participants in 45 studies) arguing that they are adequate proxies for novice analysts [67]. However, using students has also been criticized for posing threats to the generalizability of the findings [68]. Apart from the varied level of motivation of practitioners and students in participating in such experiments, using students may pose difficulties in testing some influential personal factors, such as the modeling expertise, field experience, domain familiarity, and professional background, due to a certain level of uniformity in student populations. In addition, our analysis showed that around one-third of the studies were conducted with fewer than 35 participants. Although there is no commonly accepted rule on the number of participants required in such empirical works, enlarging the respondents' base will increase the validity and generalizability of the studies. In particular, conducting experiments with industry practitioners will help in gaining a better understanding of the factors and in yielding results that are applicable and more appropriate for its intended audience.

The review of the literature also reveals a lack of empirical works on a number of potentially influential understandability factors. For instance, the effect of the medium used to present the models to their audience has not been addressed in the literature. Although the paper is usually the preferred means for interacting with model readers in practice [43], the models are typically designed using software applications, and communicated through an online environment, such as web portal and company intranet across the organization and beyond. Therefore, it is important to explore if using paper or a computer environment has any effect on model understandability. Several additional features that the computer environment brings (such as information filtering/hiding, pop-up views and animation) each with hypothetically different influence makes this factor more challenging yet interesting to investigate.

\section{Conclusion}

Our study surveyed the existing research on the process model understandability and provides an overview of the state-of-the-art in this topic. Researchers and practitioners should consider our study as a comprehensive source that offers pointers on the factors investigated in the literature and a basis for future research in this field. Our findings identify several gaps where there is a potential for major contributions. The practitioners, who aim to generate understandable process models will benefit from our findings synthesized from the existing research in this field.

The thorough analysis of the results of the studies in the field shows that we still have limited knowledge on the factors contributing to understandable process models. The studies on factors influencing process model understandability need to grow in maturity with more empirical studies [65].

Our study has various limitations mainly with regard to the underlying research method - in particular, due to the inclusion and exclusion criteria constructed and used in our systematic literature review. Our study relies on certain types of publications in reviewing the academic literature. Studies that are published as 
(non-academic) books and grey literature (technical reports, white papers, work in progress, publications without bibliographic information, unpublished publications) were not included in this study. Limitations also exist due to the search string, and inclusion and exclusion criteria used in selecting works. The study might be missing relevant works reported in academic theses or those that did not appear in the search results due to the search string that we applied in digital libraries. Similarly, we limited ourselves to publications written in English. Important and relevant studies that are published in languages other than English might be missed. These limitations are in line with the exclusion criteria of this study, but pose risks for its completeness and for the validity of the results.

\section{Appendix-A. List of primary studies included in the systematic literature review}

[S1] Agarwal R, De P, Sinha AP (1999) Comprehending Object and Process Models: An Empirical Study. IEEE Trans Softw Eng 25:541-556. doi: 10.1109/32.799953

[S2] Anda B, Sjoberg D, Jorgensen M (2001) Quality and Understandability of Use Case Models. In: Knudsen JL (ed) 15th European Conference on Object-Oriented Programming, ECOOP 2001. Springer Berlin Heidelberg, Budapest, Hungary, pp 402-428

[S3] Cruz-Lemus JA, Genero M, Manso ME, et al (2009) Assessing the understandability of UML statechart diagrams with composite states-A family of empirical studies. Empir Softw Eng 14:685-719. doi: 10.1007/s10664-009-9106-Z

[S4] Cruz-Lemus JA, Genero M, Piattini M, Toval A (2005) An Empirical Study of the Nesting Level of Composite States Within UML Statechart Diagrams. In: Akoka J, Liddle SW, Song I-Y, et al. (eds) Perspectives in Conceptual Modeling - ER 2005 Workshops AOIS, BP-UML, CoMoGIS, eCOMO, and QoIS, Klagenfurt, Austria, October 24-28, 2005. Proceedings. Springer Berlin Heidelberg, pp 1222

[S5] Cruz-Lemus JA, Maes A, Genero M, et al (2010) The impact of structural complexity on the understandability of UML statechart diagrams. Inf Sci (Ny) 180:2209-2220. doi: 10.1016/j.ins.2010. 01.026

[S6] Dumas M, Rosa M La, Mendling J, et al (2012) Understanding Business Process Models: The Costs and Benefits of Structuredness. In: Ralyte J, Franch X, Brinkkemper S, Wrycza S (eds) Advanced Information Systems Engineering 24th International Conference, CAiSE 2012, Gdansk, Poland, June 25-29, 2012. Proceedings. Springer Berlin Heidelberg, pp 31-46

[S7] Figl K, Koschmider A, Kriglstein S (2013a) Visualising Process Model Hierarchies. In: 21st European Conference on Information Systems. p Paper 180

[S8] Figl K, Laue R (2011) Cognitive Complexity in Business Process Modeling. In: Mouratidis $\mathrm{H}$, Rolland $\mathrm{C}$ (eds) Advanced Information Systems Engineering 23rd International Conference, CAiSE 2011, London, UK, June 20-24, 2011. Proceedings. Springer Berlin Heidelberg, pp 452-466

[S9] Figl K, Mendling J, Strembeck M (2013b) The Influence of Notational Deficiencies on Process Model Comprehension. J Assoc Inf Syst 14:312-338.

[S10] Figl K, Recker J, Mendling J (2013c) A study on the effects of routing symbol design on process model comprehension. Decis Support Syst 54:1104-1118. doi: 10.1016/j.dss.2012.10.037

[S11] Garcia F, Piattini M, Ruiz F, Visaggio CA (2005) Maintainability of Software Process Models: An Empirical Study. In: Ninth European Conference on Software Maintenance and Reengineering, 2005. IEEE, pp 246-255

[S12] Genero M, Miranda D, Piattini M (2003) Defining Metrics for UML Statechart Diagrams in a Methodological Way. In: Jeusfeld MA, Pastor O (eds) Conceptual Modeling for Novel Application Do- mains - ER 2003 Workshops ECOMO, IWCMQ AOIS, and XSDM, Chicago, IL, USA, October 13, 2003. Proceedings. Springer Berlin Heidelberg, pp 118-128

[S13] Glezer C, Last M, Nachmany E, Shoval P (2005) Quality and comprehension of UML interaction diagrams-an experimental comparison. Inf Softw Technol 47:675-692. doi: 10.1016/j.infsof. 2005.01.003

[S14] Gross A, Doerr J (2009) EPC vs. UML Activity Diagram - Two Experiments Examining their Usefulness for Requirements Engineering. In: 17th IEEE International Requirements Engineering Conference, 2009. RE ' 09. IEEE, pp 47-56

[S15] Hadar I, Reinhartz-Berger I, Kuflik T, et al (2013) Comparing the comprehensibility of requirements models expressed in Use Case and Tropos: Results from a family of experiments. Inf Softw Technol 55:1823-1843. doi: 10.1016/j.infsof.2013.05.003

[S16] Haisjackl C, Zugal S (2014) Investigating Differences between Graphical and Textual Declarative Process Models. In: Iliadis L, Papazoglou M, Pohl K (eds) 26th International Conference on Advanced Information Systems Engineering. Springer International Publishing, pp 194-206

[S17] Koschmider A, Kriglstein S, Ullrich M (2013) Investigations on User Preferences of the Alignment of Process Activities, Objects and Roles. In: Meersman R, Panetto H, Dillon T, et al. (eds) On the Move to Meaningful Internet Systems: OTM 2013 Conferences Confederated International Conferences: CoopIS, DOA-Trusted Cloud, and ODBASE 2013, Graz, Austria, September 9-13, 2013. Proceedings. Springer Berlin Heidelberg, pp 57-74

[S18] Mendling J, Reijers HA, Recker J (2010) Activity labeling in process modeling: Empirical insights and recommendations. Inf Syst 35:467-482. doi: 10.1016/j.is.2009.03.009

[S19] Mendling J, Strembeck M, Recker J (2012) Factors of process model comprehension-Findings from a series of experiments. Decis Support Syst 53:195-206. doi: 10.1016/j.dss.2011.12.013

[S20] Mturi E, Johannesson P (2014) A context-based process semantic annotation model for a process model repository. Bus Process Manag J 19:404-430. doi: 10.1108/14,637,151,311,319,888

[S21] Otero MC, Dolado JJ (2005) An empirical comparison of the dynamic modeling in OML and UML. J Syst Softw 77:91-102. doi: $10.1016 /$ j.jss.2004.11.022

[S22] Otero MC, Dolado JJ (2004) Evaluation of the comprehension of the dynamic modeling in UML. Inf Softw Technol 46:35-53. doi: 10.1016/S0950-5849(03)00,108-3

[S23] Ottensooser A, Fekete A, Reijers HA, et al (2012) Making sense of business process descriptions: An experimental comparison of graphical and textual notations. J Syst Softw 85:596-606. doi: 10.1016/j.jss.2011.09.023

[S24] Peleg M, Dori D (2000) The Model Multiplicity Problem: Experimenting with Real-Time Specification Methods. IEEE Trans Softw Eng 26:742-759. doi: 10.1109/32.879812

[S25] Pichler P, Weber B, Zugal S, et al (2012) Imperative versus Declarative Process Modeling Languages: An Empirical Investigation. In: Daniel F, Barkaoui K, Dustdar S (eds) Business Process Management Workshops - BPM 2011 International Workshops, Clermont-Ferrand, France, August 29, 2011, Revised Selected Papers, Part I. Springer Berlin Heidelberg, pp 383-394

[S26] Purchase HC, Colpoys L, McGill M, Carrington D (2002) UML collaboration diagram syntax: an empirical study of comprehension. In: Proceedings of the First International Workshop on Visualizing Software for Understanding and Analysis (VISSOFT 02). IEEE, Paris, France, pp 13-22

[S27] Recker J (2012) Empirical investigation of the usefulness of Gateway constructs in process models. Eur J Inf Syst 22:673689. doi: $10.1057 /$ ejis.2012.50

[S28] Recker J, Dreiling A (2007) Does It Matter Which Process Modelling Language We Teach or Use? An Experimental Study on Understanding Process Modelling Languages without Formal Edu- 
cation. In: 18th Australasian Conference on Information Systems. Toowoomba, Australia, pp 356-366

[S29] Recker J, Dreiling A (2011) The Effects of Content Presentation Format and User Characteristics on Novice Developers' Understanding of Process Models. Commun Assoc Inf Syst 28:65-84.

[S30] Recker J, Reijers HA, van de Wouw SG (2014) Process Model Comprehension: The Effects of Cognitive Abilities, Learning Style, and Strategy. Commun Assoc Inf Syst 34:199-222.

[S31] Reggio G, Ricca F, Scanniello G, et al (2013) On the comprehension of workflows modeled with a precise style: results from a family of controlled experiments. Softw Syst Model 1-24. doi: 10.1007/s10270-013-0386-9

[S32] Reijers HA, Freytag T, Mendling J, Eckleder A (2011a) Syntax highlighting in business process models. Decis Support Syst 51:339-349. doi: 10.1016/j.dss.2010.12.013

[S33] Reijers HA, Mendling J (2011) A Study Into the Factors That Influence the Understandability of Business Process Models. IEEE Trans Syst Man Cybern 41:449-462.

[S34] Reijers HA, Mendling J, Dijkman RM (2011b) Human and automatic modularizations of process models to enhance their comprehension. Inf Syst 36:881-897. doi: 10.1016/j.is.2011.03.003

[S35] Rolon E, Cardoso J, Garcia F, et al (2009) Analysis and Validation of Control-Flow Complexity Measures with BPMN Process Models. In: Halpin T, Krogstie J, Nurcan S, et al. (eds) Enterprise, Business-Process and Information Systems Modeling - 10th International Workshop, BPMDS 2009, and 14th International Conference, EMMSAD 2009, held at CAiSE 2009, Amsterdam, The Netherlands, June 8-9, 2009. Proceedings. Springer Berlin Heidelberg, pp 58-70

[S36] Sanchez-Gonzalez L, Garcia F, Mendling J, Ruiz F (2010) Prediction of Business Process Model Quality Based on Structural Metrics. In: Parsons J, Saeki M, Shoval P, et al. (eds) Conceptual Modeling - ER 2010 - 29th International Conference on Conceptual Modeling, Vancouver, BC, Canada, November 1-4, 2010. Proceedings. Springer Berlin Heidelberg, pp 458-463

[S37] Sanchez-Gonzalez L, Garcia F, Ruiz F, Mendling J (2012) Quality indicators for business process models from a gateway complexity perspective. Inf Softw Technol 54:1159-1174. doi: 10. 1016/j.infsof.2012.05.001

[S38] Sarshar K, Loos P (2005) Comparing the Control-Flow of EPC and Petri Net from the End-User Perspective. In: van der Aalst WMP, Benatallah B, Casati F, Curbera F (eds) 3rd International Conference on Business Process Management. Springer Berlin Heidelberg, Nancy, France, pp 434-439

[S39] Stitzlein C, Sanderson P, Indulska M (2013) Understanding healthcare processes: An evaluation of two process model notations. In: Human Factors and Ergonomics Society 57th Annual Meeting. pp 240-244

[S40] Storrle H (2011) On the Impact of Layout Quality to Understanding UML Diagrams. In: 2011 IEEE Symposium on Visual Languages and Human-Centric Computing (VL/HCC). IEEE, Pittsburgh, PA, pp 135-142

[S41] Storrle H (2012) On the Impact of Layout Quality to Understanding UML Diagrams: Diagram Type and Expertise. In: 2012 IEEE Symposium on Visual Languages and Human-Centric Computing (VL/HCC). IEEE, Innsbruck, pp 49-56

[S42] Storrle H (2014) On the Impact of Layout Quality to Understanding UML Diagrams: Size Matters. In: Dingel J, Schulte W, Ramos I, et al. (eds) ACM/IEEE 17th International Conference on Model Driven Engineering Languages and Systems. Springer International Publishing, pp 518-534

[S43] Weitlaner D, Guettinger A, Kohlbacher M (2013) Intuitive Comprehensibility of Process Models. In: Fischer H, Schneeberger J (eds) 5th International Conference on Subject-Oriented Business Process Management. Springer Berlin Heidelberg, pp 52-71
[S44] Yue T, Briand LC, Labiche Y (2013) Facilitating the Transition from Use Case Models to Analysis Models: Approach and Experiments. ACM Trans Softw Eng Methodol 22:1-38. doi: 10.1145/ $2,430,536.2430539$

[S45] Zugal S, Soffer P, Haisjackl C, et al (2013) Investigating expressiveness and understandability of hierarchy in declarative business process models. Softw Syst Model 14:1081-1103. doi: 10.1007/s10270-013-0356-2

\section{References}

[1] M. Dumas, M. La Rosa, J. Mendling, H.A. Reijers, Fundamentals of Business Process Management, Springer, 2013

[2] J. Pinggera, The Process of Process Modeling, University of Innsbruck, 2014.

[3] J. Recker, M. Rosemann, M. Indulska, P. Green, Business process modeling- a comparative analysis, J. Assoc. Inf. Syst. 10 (2009) 333-363.

[4] H.A. Reijers, J. Mendling, A study into the factors that influence the understandability of business process models, IEEE Trans. Syst. Man. Cybern. 41 (2011) 449-462.

[5] C. Houy, P. Fettke, P. Loos, On the theoretical foundations of research into the understandability of business process models, 22nd Eur. Conf. Inf. Syst., 2014.

[6] H.A. Reijers, J. Recker, S.G. van de Wouw, An integrative framework of the factors affecting process model understanding: a learning perspective, in: 16th Am. Conf. Inf. Syst., 2010, pp. 12-15.

[7] O.I. Lindland, G. Sindre, A. Solvberg, Understanding quality in conceptual modeling, IEEE Softw. (1994) 42-49, doi:10.1109/52.268955.

[8] H.J. Nelson, G. Poels, M. Genero, M. Piattini, A conceptual modeling quality framework, Softw. Qual. J. 20 (2012) 201-228, doi:10.1007/s11219-011-9136-9.

[9] H.A. Reijers, J. Mendling, J. Recker, Business process quality management, in: J. vom Brocke, M. Rosemann (Eds.), Handb. Bus. Process Manag. 1 Introd Methods Inf. Syst., Springer Berlin Heidelberg, 2010, pp. 167-185, doi:10.1007| 978-3-642-00416-2.

[10] S. Overhage, D.Q. Birkmeier, S. Schlauderer, Quality marks, metrics, and measurement procedures for business process models, Bus. Inf. Syst. Eng. 4 (2012) 229-246, doi:10.1007/s12599-012-0230-8.

[11] A.S. Guceglioglu, O. Demirors, A process based model for measuring process quality attributes, in: EuroSPI, Springer, Berlin Heidelberg, 2005, pp. 118-129, doi: $10.1007 / 11586012 \_12$.

[12] A.S. Guceglioglu, O. Demirors, Using software quality characteristics to measure business process quality, in: 3rd Int. Conf. Bus. Process Manag., Springer Berlin Heidelberg, Nancy, France, 2005, pp. 374-379, doi:10.1007/11538394_26.

[13] J. Becker, M. Rosemann, C. Von Uthmann, Guidelines of business process modeling, in: W. van der Aalst, J. Desel, A. Oberweis (Eds.), Bus. Process Manag. - Model. Tech. Empir. Stud., Springer Berlin Heidelberg, 2000, pp. 30-49, doi:10.1007/3-540-45594-9_3.

[14] O. Turetken, O. Demirors, Plural: a decentralized business process modeling method, Inf. Manage. 48 (2011) 235-247, doi:10.1016/j.im.2011.06.001.

[15] O. Turetken, O. Demirors, Business process modeling pluralized, in: 5th Int Conf. Subj. Bus. Process Manag., Springer Berlin Heidelberg, Deggendorf, Germany, 2013, pp. 34-51, doi:10.1007/978-3-642-36754-0 3.

[16] M. Rosemann, Potential pitfalls of process modeling: part A, Bus. Process Manage. J. 12 (2006) 249-254, doi:10.1108/14637150610657567.

[17] J. Mendling, Empirical studies in process model verification, in: K. Jensen, W. van der Aalst (Eds.), Trans. Petri Nets Other Model. Concurr. II, 2009, pp. 208-224, doi:10.1007/978-3-642-00899-3_12.

[18] I.M.-M. de Oca, M. Snoeck, H.A. Reijers, A. Rodriguez-Morffi, A systematic literature review of studies on business process modeling quality, Inf. Softw. Technol. 58 (2015) 187-205, doi:10.1016/j.infsof.2014.07.011.

[19] L.S. Gonzalez, F.G. Rubio, F.R. Gonzalez, M.P. Velthuis, Measurement in business processes: a systematic review, Bus. Process Manage. J. 16 (2010) 114-134, doi:10.1108/14637151011017976.

[20] C. Houy, P. Fettke, P. Loos, Understanding understandability of conceptual models: what are we actually talking about? in: Int. Conf. Concept. Model. ER-2012, Springer, 2012, pp. 64-77, doi:10.1007/978-3-642-34002-4_5.

[21] J. Mendling, H.A. Reijers, W.M.P. van der Aalst, Seven process modeling guidelines (7PMG), Inf. Softw. Technol. 52 (2010) 127-136, doi:10.1016/j.infsof.2009. 08.004 .

[22] M. Schrepfer, Modeling Guidelines for Business Process Models, Humboldt-Universitat zu Berlin, 2010.

[23] B. Kitchenham, S. Charters, Guidelines for performing systematic literature reviews in software engineering, Tech. rep., Technical report, EBSE Technical Report EBSE-2007-01, 2007.

[24] J. Webster, R.T. Watson, Analyzing the past to prepare for the future: writing a literature review, MIS Q. 26 (2002) xiii-xxiii.

[25] C. Glezer, M. Last, E. Nachmany, P. Shoval, Quality and comprehension of UML interaction diagrams-an experimental comparison, Inf. Softw. Technol. 47 (2005) 675-692, doi:10.1016/j.infsof.2005.01.003.

[26] Object Management Group, OMG UML Version 2.5, (2015). doi:10.1007/ s002870050092.

[27] N.A. Karagoz, O. Demirors, Conceptual modeling notations and techniques, in: S. Robinson, R. Brooks, K. Kotiadis, D.-J. van der Zee (Eds.), Concept. Model. Discret. Simul., CRC Press, 2010, pp. 179-209, doi:10.1201/9781439810385-c7. 
[28] C. Wohlin, Guidelines for snowballing in systematic literature studies and a replication in software engineering, 18th Int. Conf. Eval. Assess. Softw. Eng., ACM, New York, New York, USA, 2014, doi:10.1145/2601248.2601268.

[29] A. Gemino, Y. Wand, A framework for empirical evaluation of conceptual modeling techniques, Requir. Eng. 9 (2004) 248-260, doi:10.1007/ s00766-004-0204-6.

[30] A. Burton-Jones, Y. Wand, R. Weber, Guidelines for empirical evaluations of conceptual modeling grammars, J. Assoc. Inf. Syst. 10 (2009) 495-532.

[31] P.A. Kirschner, Cognitive load theory: implications of cognitive load theory on the design of learning, Learn. Instr. 12 (2002) 1-10, doi:10.1016/ S0959-4752(01)00014-7.

[32] S. Zugal, J. Pinggera, H. Reijers, M. Reichert, B. Weber, Making the case for measuring mental effort, in: 2nd Int. Work. Exp. Empir. Stud. Softw. Model. EESSMod 2012, ACM Press, Innsbruck, Austria, 2012, pp. 37-42, doi:10.1145/ 2424563.2424571.

[33] F.D. Davis, Perceived usefulness, perceived ease of use, and user acceptance of information technology, MIS Q. 13 (1989) 319-340, doi:10.2307/249008.

[34] V. Venkatesh, M.G. Morris, G.B. Davis, F.D. Davis, User acceptance of information technology: toward a unified view, MIS Q. 27 (2003) 425-478.

[35] D.L. Moody, The method evaluation model: a theoretical model for validating information systems design methods, in: 11th Eur. Conf. Inf. Syst., Naples, Italy, 2003, pp. 1311-1326.

[36] J. Recker, M. Rosemann, P. Green, M. Indulska, Do ontological deficiencies in modeling grammars matter? MIS 0. 35 (2011) 57-79.

[37] E. Rolon, J. Cardoso, F. Garcia, F. Ruiz, M. Piattini, Analysis and validation of control-flow complexity measures with BPMN process models, in: 10th Int. Work. Bus. Process Model. Dev. Support, Springer Berlin Heidelberg, Amsterdam, The Netherlands, 2009, pp. 58-70, doi:10.1007/978-3-642-01862-6 6 .

[38] J. Mendling, H.M.W. Verbeek, B.F. van Dongen, W.M.P. van der Aalst, G. Neumann, Detection and prediction of errors in EPCs of the SAP reference model, Data Knowl. Eng. 64 (2008) 312-329, doi:10.1016/j.datak.2007.06.019.

[39] R. Laue, J. Mendling, Structuredness and its significance for correctness of process models, Inf. Syst. e-Bus. Manage. 8 (2010) 287-307, doi:10.1007/ s10257-009-0120-X

[40] H.A. Reijers, J. Mendling, Modularity in process models: review and effects, in: 6th Int. Conf. Bus. Process Manage., Springer Berlin Heidelberg, Milan, Italy, 2008, pp. 20-35, doi:10.1007/978-3-540-85758-7_5.

[41] D.L. Moody, Cognitive load effects on end user understanding of conceptual models: an experimental analysis, in: 8th East Eur. Conf. Adv. Databases Inf. Syst., Springer Berlin Heidelberg, Budapest, Hungary, 2004, pp. 129-143, doi:10.1007/978-3-540-30204-9_9.

[42] W.M.P. van der Aalst, M. Pesic, DecSerFlow: towards a truly declarative service flow language, in: M. Bravetti, M. Nunez, G. Zavaratto (Eds.), 3rd Int. Work. Web Serv. Form. Methods, Springer Berlin Heidelberg, Vienna, Austria, 2006, pp. 1-23, doi:10.1007/11841197_1.

[43] H.A. Reijers, J. Mendling, R.M. Dijkman, Human and automatic modularizations of process models to enhance their comprehension, Inf. Syst. 36 (2011) 881 897, doi:10.1016/j.is.2011.03.003.

[44] S. Zugal, P. Soffer, J. Pinggera, B. Weber, Expressiveness and understandability considerations of hierarchy in declarative business process models, in: 13th Int. Conf. Bus. Process Model. Dev. Support, Springer Berlin Heidelberg, Gdansk, Poland, 2012, pp. 167-181, doi:10.1007/978-3-642-31072-0 12.

[45] K. Figl, A. Koschmider, S. Kriglstein, Visualising process model hierarchies, in: 21st Eur. Conf. Inf. Syst., 2013, p. 180.

[46] J. Sweller, Cognitive load theory, learning difficulty, and instructional design, Learn. Instr. 4 (1994) 295-312, doi:10.1016/0959-4752(94)90003-5.

[47] P. Harmon, C. Wolf, The state of business process management 2014, 2014 http://ww.bptrends.com/.

[48] A. Nigam, N.S. Caswell, Business artifacts: an approach to operational specification, IBM Syst. J. 42 (2003) 428-445, doi:10.1147/sj.423.0428.
[49] V. Bernstein, P. Soffer, Identifying and quantifying visual layout features of business process models, in: K. Gaaloul, R Schmidt, S. Nurcan, S. Guerreiro, Q. Ma (Eds.), 16th Int. Conf. Bus. Process Model. Dev. Support, Springer International Publishing, Stockholm, Sweden, 2015, pp. 200-213, doi:10.1007| 978-3-319-19237-6_13.

[50] O. Kilic, B. Say, O. Demirors, An experimental study on the cognitive characteristics of modeling notations, in: F.V.C. Ficarra (Ed.), Adv. Dyn. Static Media Interact. Syst., Blue Herons Editions, 2011.

[51] W. Bandara, G.G. Gable, M. Rosemann, Factors and measures of business process modelling: model building through a multiple case study, Eur. J. Inf. Syst. 14 (2005) 347-360, doi:10.1057/palgrave.ejis.3000546.

[52] J. Recker, Continued use of process modeling grammars: the impact of individual difference factors, Eur. J. Inf. Syst. 19 (2010) 76-92, doi:10.1057/ejis.2010.5.

[53] J. Mendling, M. Strembeck, J. Recker, Factors of process model comprehension-findings from a series of experiments, Decis. Support Syst. 53 (2012) 195-206, doi:10.1016/j.dss.2011.12.013.

[54] R. Kanfer, P.L. Ackerman, T.C. Murtha, B. Dugdale, L. Nelson, Goal setting, conditions of practice, and task performance: a resource allocation perspective, J. Appl. Psychol. 79 (1994) 826-835, doi:10.1037/0021-9010.79.6.826.

[55] J.B. Gassen, J. Mendling, L.H. Thom, J.P.M. de Oliveira, Towards guiding process modelers depending upon their expertise levels, in: A. Persson, J. Stirna (Eds.), CAiSE 2015 Int. Work., Springer International Publishing, Stockholm, Sweden, 2015, pp. 69-80, doi:10.1007/978-3-319-19243-76.

[56] J. Bennedsen, M.E. Caspersen, Abstraction ability as an indicator of success for learning object-oriented programming? ACM SIGCSE Bull. 38 (2006) 39-43, doi:10.1145/1138403.1138430.

[57] W. Winn, An account of how readers search for information in diagrams, Contemp. Educ. Psychol. 18 (1993) 162-185, doi:10.1006/ceps.1993.1016.

[58] R.M. Felder, L.K. Silverman, Learning and teaching styles in engineering education, Eng. Educ. 78 (1988) 674-681, doi:10.1109/FIE.2008.4720326.

[59] R.M. Felder, R. Brent, Understanding student differences, J. Eng. Educ. 94 (2005) 57-72.

[60] D. Kember, J. Biggs, D.Y.P. Leung, Examining the multidimensionality of approaches to learning through the development of a revised version of the Learning Process Questionnaire, Br. J. Educ. Psychol. (2004) 261-280, doi:10. $1348 / 000709904773839879$

[61] A. Lakhotia, Understanding someone else's code: analysis of experiences, J. Syst. Softw. 23 (1993) 269-275, doi:10.1016/0164-1212(93)90101-3.

[62] R.E. Mayer, Multimedia Learning, Cambridge University Press, 2001 http://dx. doi.org/10.1017/CB09781139164603.

[63] R. Laue, A. Gadatsch, Measuring the understandability of business process models - are we asking the right questions? in: BPM 2010 Int. Work. Educ. Track, Springer Berlin Heidelberg, Hoboken, NJ, USA, 2010, pp. 37-48, doi:10. 1007/978-3-642-20511-8_4.

[64] D.I.K. Sjoberg, J.E. Hannay, O. Hansen, V.B. Kampenes, A. Karahasanovic, N.K. Liborg, A.C. Rekdal, A survey of controlled experiments in software engineering, IEEE Trans. Softw. Eng. 31 (2005) 733-753, doi:10.1109/TSE.2005.97.

[65] J. Recker, J. Mendling, The state of the art of business process management research as published in the BPM conference, Bus. Inf. Syst. Eng. (2015) 1-18, doi:10.1007/s12599-015-0411-3.

[66] J. Mendling, H.A. Reijers, J. Cardoso, What makes process models understandable? in: 5th Int. Conf. Bus. Process Manage., Springer Berlin Heidelberg, Brisbane, Australia, 2007, pp. 48-63, doi:10.1007/978-3-540-75183-0_4.

[67] A. Burton-Jones, P. Meso, The effects of decomposition quality and multiple forms of information on novices' understanding of a domain from a conceptual model, J. Assoc. Inf. Syst. 9 (2008) 784-802.

[68] C.D. Kam, J.R. Wilking, E.J. Zechmeister, Beyond the "Narrow Data Base": another convenience sample for experimental research, Political Behav. 29 (2007) 415-440, doi:10.1007/s11109-007-9037-6. 